TRANSACTIONS OF THE

AMERICAN MATHEMATICAL SOCIETY

Volume 352, Number 11, Pages 5093-5137

S 0002-9947(00)02630-1

Article electronically published on March 16, 2000

\title{
HOMOLOGY MANIFOLD BORDISM
}

\author{
HEATHER JOHNSTON AND ANDREW RANICKI
}

\begin{abstract}
The Bryant-Ferry-Mio-Weinberger surgery exact sequence for compact $A N R$ homology manifolds of dimension $\geq 6$ is used to obtain transversality, splitting and bordism results for homology manifolds, generalizing previous work of Johnston.

First, we establish homology manifold transversality for submanifolds of dimension $\geq 7$ : if $f: M \rightarrow P$ is a map from an $m$-dimensional homology manifold $M$ to a space $P$, and $Q \subset P$ is a subspace with a topological $q$-block bundle neighborhood, and $m-q \geq 7$, then $f$ is homology manifold $s$-cobordant to a map which is transverse to $Q$, with $f^{-1}(Q) \subset M$ an $(m-q)$-dimensional homology submanifold.

Second, we obtain a codimension $q$ splitting obstruction $s_{Q}(f) \in L S_{m-q}(\Phi)$ in the Wall $L S$-group for a simple homotopy equivalence $f: M \rightarrow P$ from an $m$-dimensional homology manifold $M$ to an $m$-dimensional Poincaré space $P$ with a codimension $q$ Poincaré subspace $Q \subset P$ with a topological normal bundle, such that $s_{Q}(f)=0$ if (and for $m-q \geq 7$ only if) $f$ splits at $Q$ up to homology manifold $s$-cobordism.

Third, we obtain the multiplicative structure of the homology manifold bordism groups $\Omega_{*}^{H} \cong \Omega_{*}^{T O P}\left[L_{0}(\mathbb{Z})\right]$.
\end{abstract}

\section{INTRODUCTION}

Homology manifolds are spaces with the local homology properties of topological manifolds, but not necessarily their geometric properties such as transversality. The results of Johnston [6] on the bordism and transversality properties of highdimensional homology manifolds are extended here using the methods of surgery theory. The extent to which transversality holds in a homology manifold is a measure of how close it is to being a topological manifold. It is not possible to investigate transversality in homology manifolds by direct geometric methods, so as in [6] we employ bordism and surgery instead.

We start with a brief recollection of transversality for differentiable manifolds. Suppose that $P^{n}$ is an $n$-dimensional differentiable manifold and $Q^{n-q} \subset P^{n}$ is a codimension $q$ submanifold with a $q$-plane normal bundle

$$
\nu_{Q \subset P}: Q \rightarrow B O(q) .
$$

Received by the editors March 25, 1998.

2000 Mathematics Subject Classification. Primary 57P05, Secondary 19 J25.

Key words and phrases. Homology manifolds, bordism, transversality, surgery.

This work was carried out in connection with the first named author's EPSRC Visiting Fellowship in Edinburgh in August, 1997. 
A smooth map $f: M \rightarrow P$ from an $m$-dimensional differentiable manifold $M^{m}$ is transverse to $Q \subset P$ if the inverse image of $Q$ is a codimension $q$ submanifold

$$
N^{m-q}=f^{-1}(Q) \subset M^{m}
$$

with normal $q$-plane bundle the pullback of $\nu_{Q \subset P}$ along $g=f \mid: N \rightarrow Q$

$$
\nu_{N \subset M}=g^{*} \nu_{Q \subset P}: N \rightarrow B O(q) .
$$

The classical result of Thom is that every map $f: M^{m} \rightarrow P^{n}$ is homotopic (by a small homotopy) to a smooth map which is transverse to $Q \subset P$. This result was proved by direct analytic methods.

Topological manifolds also have transversality, but the proof is very indirect, relying heavily on surgery theory - see Kirby and Siebenmann [8] (III,§1), Marin 10] and Quinn [13. Instead of vector bundles it is necessary to work with normal microbundles, although we shall use the formulation in terms of the topological block bundles of Rourke and Sanderson [17].

The essential aspect of transversality is that a submanifold has a nice normal (vector or block) bundle, as formalized in the following definition.

Definition. A codimension $q$ bundle subspace $(Q, R, \xi)(q \geq 1)$ of a space $P$ is a subspace $Q \subset P$ together with a topological $q$-block bundle

$$
\left(D^{q}, S^{q-1}\right) \rightarrow(E(\xi), S(\xi)) \rightarrow Q
$$

such that

$$
P=E(\xi) \cup_{S(\xi)} R,
$$

where $R=\overline{P \backslash E(\xi)}$. When $R$ and $\xi$ are clear we say that $Q$ is a codimension $q$ bundle subspace of $P$.

Topological $q$-block bundles over a space $Q$ are classified by the homotopy classes of maps from $Q$ to a classifying space $\overparen{B T O P}(q)$, so we write such a bundle $\xi$ as a map

$$
\xi: Q \rightarrow \widetilde{B O P}(q)
$$

If $P$ is an $n$-dimensional topological manifold and $Q \subset P$ is a triangulable locally flat codimension $q$ submanifold with $n-q \geq 5$ or $q \leq 2$, then $Q$ is a codimension $q$ bundle subspace of $P$ with

$$
\xi=\nu_{Q \subset P}: Q \rightarrow \widetilde{\operatorname{TOP}(q)}
$$

a normal topological $q$-block bundle, by Theorem 4.9 of Rourke and Sanderson [17]. (Hughes, Taylor and Williams [5] obtained a topological regular neighborhood theorem for arbitrary locally flat submanifolds in a manifold of dimension $\geq 5$, in which the neighborhood is the mapping cylinder of a manifold approximate fibration).

In the applications of codimension $q$ bundle subspaces $(Q, R, \xi) \subset P$ we shall only be concerned with the case when $P$ is a finite $C W$ complex and $Q, R \subset P$ are subcomplexes.

Definition (Submanifold transversality). Let $P$ be a space with a codimension $q$ bundle subspace $(Q, R, \xi)$.

(i) A map $f: M \rightarrow P$ from an $m$-dimensional manifold $M$ is transverse to $Q \subset P$ if the inverse image of $Q$

$$
N^{m-q}=f^{-1}(Q) \subset M^{m}
$$


is a locally flat codimension $q$ submanifold with the pullback normal bundle.

(ii) A map $f: M \rightarrow P$ is $s$-transverse to $Q \subset P$ if it is $s$-cobordant to a transverse map.

Of course, the submanifolds of the manifold $M$ and bundles in the above definitions are understood to be in the same category as $M$ itself. For simplicity, we shall only be considering compact oriented manifolds.

An $m$-dimensional homology manifold $M$ is a finite-dimensional $A N R$ such that for each $x \in M$

$$
H_{r}(M, M \backslash\{x\})= \begin{cases}\mathbb{Z} & \text { if } r=m, \\ 0 & \text { if } r \neq m\end{cases}
$$

We shall say that an $m$-dimensional homology manifold $M$ has codimension $q$ $s$-transversality if every map $f: M \rightarrow P$ is $s$-transverse to every codimension $q$ bundle subspace $Q \subset P$. (It is unknown if the analogue of the topological $s$ cobordism theorem holds for homology manifolds).

An $m$-dimensional homology manifold $M$ is resolvable if there exists a $C E$ map $h: M_{T O P} \rightarrow M$ from an $m$-dimensional topological manifold $M_{T O P}$. (Roughly speaking, a $C E$ map is a map with contractible point inverses). Resolvable homology manifolds have codimension $q$ s-transversality for all $q \geq 1:$ if $f: M \rightarrow P$ is a map from a resolvable $m$-dimensional homology manifold and $Q \subset P$ is a codimension $q$ bundle subspace, then the mapping cylinder of $h$ is a homology manifold $s$-cobordism

$$
\left(g ; f, f_{T O P}\right):\left(W ; M, M_{T O P}\right) \rightarrow P
$$

from $f$ to a map $f_{T O P}: M_{T O P} \rightarrow P$ which can be made (topologically) transverse to $Q \subset P$.

Quinn [11] used controlled surgery to prove that for $m \geq 6$ an $m$-dimensional homology manifold $M$ with codimension $m s$-transversality is resolvable. The resolution obstruction of Quinn [12]

$$
i(M) \in H_{m}\left(M ; L_{0}(\mathbb{Z})\right)
$$

is such that $i(M)=0$ if (and for $m \geq 6$ only if) $M$ is resolvable; for connected $M$ the obstruction takes values in $H_{m}\left(M ; L_{0}(\mathbb{Z})\right)=\mathbb{Z}$. The invariant $i(M)$ is the obstruction to a degree 1 map $f: M^{m} \rightarrow S^{m}$ being $s$-transverse to some point $* \in S^{m}$. Bryant, Ferry, Mio and Weinberger 1] constructed exotic homology manifolds $M^{m}$ in dimensions $m \geq 6$ which are not resolvable, and initiated the surgery classification theory for high-dimensional homology manifolds up to $s$-cobordism. In Section 1 we shall modify the construction of [1] to obtain a connected homology manifold $M=N_{I}$ with prescribed resolution obstruction $I \in L_{0}(\mathbb{Z})$, starting with any connected $m$-dimensional topological manifold $N(m \geq 6)$. This homology manifold is not homotopy equivalent to $N$, but it is in a prescribed homology manifold normal bordism class of $N$.

The first-named author used the theory of [1] to prove that $m$-dimensional homology manifolds have codimension $q s$-transversality and splitting in the following cases.

Theorem (Homology manifold $\pi-\pi s$-transversality, Johnston [6]). Let $f: M \rightarrow$ $P$ be a map from an $m$-dimensional homology manifold $M$ to a space $P$ with a codimension $q$ bundle subspace $(Q, R, \xi)$, with $m-q \geq 6$. 
(i) If $q=1, \xi$ is trivial, and $R=R_{1} \sqcup R_{2}$ is disconnected with $\pi_{1}(Q) \cong \pi_{1}\left(R_{1}\right)$, then $f$ is s-transverse to $Q \subset P$.

(ii) If $q \geq 3$, then $f$ is $s$-transverse to $Q \subset P$.

Definition. (i) A codimension q Poincaré bundle subspace $(Q, R, \xi)$ of an $m$-dimensional Poincaré space $P$ is a codimension $q$ bundle subspace such that $Q$ is an $(m-q)$-dimensional Poincaré space and $(R, S(\xi))$ is an $m$-dimensional Poincaré pair, where $S(\xi)$ is the total space of the $S^{q-1}$-bundle of $\xi$ over $P$.

(ii) Let $P, Q, R, \xi$ be as in (i). A simple homotopy equivalence $f: M \rightarrow P$ from an $m$-dimensional homology manifold $M$ splits at $Q \subset P$ if $f$ is $s$-cobordant to a simple homotopy equivalence (also denoted by $f$ ) which is transverse to $Q \subset P$ and such that the restrictions $f\left|: f^{-1}(Q) \rightarrow Q, f\right|: f^{-1}(R) \rightarrow R$ are also simple homotopy equivalences.

Theorem (Homology manifold splitting, Johnston [6]). Let $f: M \rightarrow P$ be a simple homotopy equivalence from an m-dimensional homology manifold $M$ to an $m$-dimensional Poincaré space $P$ with a codimension $q$ Poincaré bundle subspace $(Q, R, \xi)$, with $m-q \geq 6$.

(i) (Codimension $1 \pi-\pi$ splitting) If $q=1, \xi$ is trivial, and $R=R_{1} \sqcup R_{2}$ is disconnected with $\pi_{1}(Q) \cong \pi_{1}\left(R_{1}\right)$, then $f$ splits at $Q \subset P$.

(ii) (Browder splitting) If $q \geq 3$, then $f$ splits at $Q \subset P$ if and only if the restriction $g=f \mid: f^{-1}(Q) \rightarrow Q$ has surgery obstruction $\sigma_{*}(g)=0 \in L_{m-q}\left(\mathbb{Z}\left[\pi_{1}(Q)\right]\right)$.

In Sections 2, 3, and 4 we shall use the theory of [1 to obtain even stronger results on homology manifold transversality and codimension $q$ splitting. The results of this paper require the slightly higher dimension hypothesis $m-q \geq 7$. The extra dimension is needed to apply codimension $1 \pi-\pi$ splitting, (i) above, to a homology manifold of dimension $m-q$. Thus we require $m-q-1 \geq 6$, or $m-q \geq 7$.

Wall [18] (Chapter 11) obtained a codimension $q$ splitting obstruction $s_{Q}(f) \in$ $L S_{m-q}(\Phi)$ for a simple homotopy equivalence $f: M \rightarrow P$ from an $m$-dimensional topological manifold $M$ to an $m$-dimensional Poincaré space $P$ with a codimension $q \geq 1$ Poincaré bundle subspace $(Q, R, \xi)$, such that $s_{Q}(f)=0$ if (and for $m-q \geq 5$ only if) $f$ splits at $Q \subset P$. Our first main result obtains the analogous obstruction for the codimension $q$ splitting of homology manifolds. (The full statement will be given in Theorem 3.2 .)

Theorem 0.1. A simple homotopy equivalence $f: M \rightarrow P=E(\xi) \cup_{S(\xi)} R$ from an $m$-dimensional homology manifold $M$ to an $m$-dimensional Poincaré space $P$ with a codimension $q$ Poincaré bundle subspace $(Q, R, \xi)$ has a codimension $q$ splitting obstruction

$$
s_{Q}(f) \in L S_{m-q}(\Phi)
$$

such that $s_{Q}(f)=0$ if (and for $m-q \geq 7$ only if) $f$ splits at $Q \subset P$.

Our second main result establishes homology manifold $s$-transversality in the case $m-q \geq 7$, generalizing the homology manifold $\pi-\pi s$-transversality theorem of 6 . This result appears as Theorem 4.1 below.

Theorem 0.2. Let $f: M \rightarrow P=E(\xi) \cup_{S(\xi)} R$ be a map from an $m$-dimensional homology manifold $M$ to a space $P$ with a codimension $q$ bundle subspace $(Q, R, \xi)$. If $m-q \geq 7$, then $f$ is s-transverse to $Q \subset P$. 
In Sections 5 and 6 we consider $s$-transversality for a map $f: M \rightarrow P$ from a homology manifold $M$ to the polyhedron $P=|K|$ of a (finite) simplicial complex $K$. Instead of seeking $s$-transversality to just one codimension $q$ bundle subspace $Q \subset P$, we consider $s$-transversality to all the dual cells $|D(\sigma, K)| \subset P(\sigma \in K)$ at once, following the work of Cohen [3] on $P L$ manifold transversality.

The dual cells of a simplicial complex $K$ are the subcomplexes of $K^{\prime}$,

$$
D(\sigma, K)=\left\{\widehat{\sigma}_{0} \widehat{\sigma}_{1} \cdots \widehat{\sigma}_{p} \in K^{\prime} \mid \sigma \leq \sigma_{0}<\sigma_{1}<\cdots<\sigma_{p}\right\}(\sigma \in K) .
$$

The boundary of a dual cell is the subcomplex

$$
\begin{aligned}
\partial D(\sigma, K) & =\bigcup_{\tau>\sigma} D(\tau, K) \\
& =\left\{\widehat{\sigma}_{0} \widehat{\sigma}_{1} \cdots \widehat{\sigma}_{p} \in K^{\prime} \mid \sigma<\sigma_{0}<\sigma_{1}<\cdots<\sigma_{p}\right\} \subset D(\sigma, K) .
\end{aligned}
$$

Definition (Dual transversality). (i) A map $f: M \rightarrow|K|$ from an $m$-dimensional manifold $M$ (in some manifold category) is dual transverse if the inverse images of the dual cells are codimension $|\sigma|$ submanifolds

$$
M(\sigma)^{m-|\sigma|}=f^{-1}(D(\sigma, K)) \subseteq M^{m}
$$

with boundary

$$
\partial M(\sigma)=f^{-1}(\partial D(\sigma, K))=\bigcup_{\tau>\sigma} M(\tau) .
$$

(ii) An $m$-dimensional manifold $M$ has dual $s$-transversality if every map $f$ : $M \rightarrow|K|$ is $s$-cobordant to a dual transverse map.

Dual transversality implies submanifold transversality : if the map

$$
f: M^{m} \rightarrow P=|K|=E(\xi) \cup_{S(\xi)} R
$$

is dual transverse, then $f$ is transverse to every polyhedral codimension $q$ bundle subspace $Q \subset P$. $P L$ manifolds and $P L$ homology manifolds $M$ have dual transversality, with every simplicial map $f: M \rightarrow K^{\prime}$ dual transverse - in this case each inverse image $f^{-1}(D(\sigma, K)) \subset M(\sigma \in K)$ is automatically a $P L$ submanifold of codimension $|\sigma|$ (Cohen [3]), so there is no need to use $s$-cobordisms. Topological manifolds $M$ have dual transversality by the work of Kirby and Siebenmann [8] and Quinn [13], with every map $f: M \rightarrow|K|$ homotopic to a dual transverse map.

The $s$-transversality result of Theorem 0.2 can be applied inductively to obtain dual $s$-transversality for a map $f: M^{m} \rightarrow|K|$ in the case when the inverse images $f^{-1}(D(\sigma, K))(\sigma \in K)$ are required to be homology manifolds of dimensions $m-$ $|\sigma| \geq 7$.

Corollary 0.3. If $f: M \rightarrow|K|$ is a map from an m-dimensional homology manifold $M$ to the polyhedron of a $k$-dimensional simplicial complex $K$ with $m-k \geq 7$, then $f$ is dual s-transverse.

On the other hand, if $m-k<7$, dual transversality may be obstructed. Consider for example the case of $f: M \rightarrow|K|$ for $m=k$, with $M$ connected. In this case the resolution obstruction is easily shown to be an obstruction to dual transversality. If $f: M \rightarrow|K|$ is dual transverse, then for some $m$-simplex $\sigma^{m} \in K$ and a sequence of faces $\sigma^{0}<\sigma^{1}<\cdots<\sigma^{m}$ the inverse images $M\left(\sigma^{j}\right)^{m-j}=f^{-1}\left(D\left(\sigma^{j}, K\right)\right)$ are non-empty codimension $j$ homology submanifolds of $M$ with

$$
M\left(\sigma^{m}\right)^{0} \subset \partial M\left(\sigma^{m-1}\right) \subset M\left(\sigma^{m-1}\right)^{1} \subset \cdots \subset M\left(\sigma^{0}\right)^{m} \subset M .
$$

Quinn $([12], 1.1)$ proved that the resolution obstruction $i(X)$ is such that: 
(i) $i(U)=i(X)$ for any open subset $U \subset X$ of a homology manifold $X$, and

(ii) $i(X)=i(\partial X)$ for any connected homology manifold $X$ with non-empty boundary $\partial X$.

It follows that

$$
i\left(M\left(\sigma^{m}\right)^{0}\right)=i\left(M\left(\sigma^{m-1}\right)^{1}\right)=\cdots=i\left(M\left(\sigma^{0}\right)^{m}\right)=i(M) .
$$

Now $M\left(\sigma^{m}\right)$ is a 0 -dimensional homology manifold, which is a (finite) union of points, so that $i(M)=i\left(M\left(\sigma^{m}\right)\right)=0$ and $M$ is resolvable. In Section 6 we use the algebraic topology of homology manifold bordism to prove a strong generalization of this result. Although the resolution obstruction continues to play a key role, the general form of the dual transversality obstruction is more complicated algebraically.

For any space $X$ let $\Omega_{m}^{H}(X)$ be the bordism group of maps $M \rightarrow X$ from $m$ dimensional homology manifolds. An $m$-dimensional topological manifold is an $m$-dimensional homology manifold, so there are evident forgetful maps

$$
\Omega_{m}^{T O P}(X) \rightarrow \Omega_{m}^{H}(X) .
$$

For any simplicial complex $K$, let $\Omega_{m}^{H, \pitchfork}(K)$ be the bordism group of dual transverse maps $M \rightarrow|K|$ from $m$-dimensional homology manifolds. Forgetting dual transversality gives maps

$$
A^{H}: \Omega_{*}^{H, \pitchfork}(K) \rightarrow \Omega_{*}^{H}(K) .
$$

The extent to which dual transversality holds for homology manifolds up to bordism is measured by the extent to which the maps $A^{H}$ are isomorphisms.

Our third main result relates the obstruction to homology manifold dual $s$ transversality to the resolution obstruction, and identifies the fibre of $A^{H}$ with a generalized homology theory. The full statement will be given in Theorem 6.6 including the following result:

Theorem 0.4. For $m \geq 6$ the $K$-transverse homology manifold bordism groups $\Omega_{m}^{H, \pitchfork}(K)$ are related to the homology manifold bordism groups $\Omega_{m}^{H}(K)$ by an exact sequence

$$
\cdots \rightarrow \Omega_{m}^{H, \pitchfork}(K) \stackrel{A^{H}}{\rightarrow} \Omega_{m}^{H}(K) \rightarrow H_{m}\left(K ; \mathcal{L}_{\bullet}\right) \rightarrow \Omega_{m-1}^{H, \pitchfork}(K) \rightarrow \ldots
$$

with $\mathcal{L}_{\bullet}$ a spectrum such that $\pi_{0}\left(\mathcal{L}_{\bullet}\right)=\mathbb{Z}\left[L_{0}(\mathbb{Z}) \backslash\{0\}\right]$ and $\pi_{m}\left(\mathcal{L}_{\bullet}\right)=0$ for $m \geq 6$.

Ferry and Pedersen 4 showed that the Spivak normal fibration $\nu_{M}$ of an $m$ dimensional homology manifold $M$ admits a canonical TOP reduction $\widetilde{\nu}_{M}$, so that there is a canonical bordism class of normal maps $M_{T O P} \rightarrow M$ from topological manifolds. The surgery obstruction $\sigma_{*}\left(M_{T O P} \rightarrow M\right) \in L_{m}\left(\mathbb{Z}\left[\pi_{1}(M)\right]\right)$ is determined by the resolution obstruction $i(M) \in H_{m}\left(M ; L_{0}(\mathbb{Z})\right)$.

For any abelian group $A$ let $A\left[L_{0}(\mathbb{Z})\right]$ be the abelian group of finite linear combinations

$$
\sum_{i \in L_{0}(\mathbb{Z})} a_{i}[i] \quad\left(a_{i} \in A\right),
$$

which is the direct sum of $L_{0}(\mathbb{Z})$ copies of $A$ :

$$
A\left[L_{0}(\mathbb{Z})\right]=\mathbb{Z}\left[L_{0}(\mathbb{Z})\right] \otimes_{\mathbb{Z}} A=\bigoplus_{L_{0}(\mathbb{Z})} A
$$


Theorem (The additive structure of homology manifold bordism, Johnston [6]). For any simplicial complex $K$ the map of bordism groups

$$
\Omega_{m}^{H}(K) \rightarrow \Omega_{m}^{T O P}(K)\left[L_{0}(\mathbb{Z})\right],(M, f) \mapsto\left(M_{T O P}, f_{T O P}\right)[i(M)],
$$

is an isomorphism for $m \geq 6$, with

$$
f_{T O P}: M_{T O P} \rightarrow M \stackrel{f}{\rightarrow}|K| .
$$

In Section 7 we shall analyze the multiplicative structure on $\Omega_{m}^{T O P}(K)\left[L_{0}(\mathbb{Z})\right]$ which corresponds to the cartesian product of homology manifolds under the isomorphism $\Omega_{m}^{H}(K) \cong \Omega_{m}^{T O P}(K)\left[L_{0}(\mathbb{Z})\right]$.

We should like to thank the referee and Bruce Hughes for helpful comments on the manuscript.

\section{Homology MANIFOLD SURGERY AND BORDISM}

We review and extend the surgery theory of $A N R$ homology manifolds.

An (oriented) simple $m$-dimensional Poincaré space $X$ is a compact $A N R$ with a fundamental class $[X] \in H_{m}(X)$ such that the chain map

$$
[X] \cap-: C(\widetilde{X})^{m-*}=\operatorname{Hom}_{\mathbb{Z}\left[\pi_{1}(X)\right]}\left(C(\tilde{X}), \mathbb{Z}\left[\pi_{1}(X)\right]\right)^{m-*} \rightarrow C(\tilde{X})
$$

is a simple $\mathbb{Z}\left[\pi_{1}(X)\right]$-module chain equivalence inducing isomorphisms

$$
[X] \cap-: H^{m-*}(\widetilde{X}) \cong H_{*}(\widetilde{X}),
$$

with $\tilde{X}$ the universal cover of $X$ and $H^{*}(\tilde{X}) \equiv H_{-*}\left(C(\widetilde{X})^{-*}\right)$. (A compact $A N R$ has a preferred simple homotopy type by the work of Chapman [2.) In particular, an $m$-dimensional homology manifold is an $m$-dimensional Poincaré space.

The manifold structure set $\mathcal{S}(X)$ of a simple $m$-dimensional Poincaré space $X$ is the set of equivalence classes of pairs $(M, h)$ with $M$ an $m$-dimensional topological manifold and $h: M \rightarrow X$ a simple homotopy equivalence, with $\left(M_{1}, h_{1}\right) \simeq\left(M_{2}, h_{2}\right)$ if there exists an $s$-cobordism $\left(W ; M_{1}, M_{2}\right)$ with a simple homotopy equivalence of the type

$$
\left(g ; h_{1}, h_{2}\right):\left(W ; M_{1}, M_{2}\right) \rightarrow X \times([0,1] ;\{0\},\{1\}) .
$$

The normal invariant set $\mathcal{T}(X)$ is the bordism set of degree 1 normal maps $(f, b)$ : $M \rightarrow X$ from topological manifolds, with $b: \nu_{M} \rightarrow \widetilde{\nu}_{X}$ a bundle map from the normal bundle of $M$ to a TOP reduction of the Spivak normal fibration of $X$. For $m \geq 5$ the Browder-Novikov-Sullivan-Wall surgery theory for topological manifolds gives the surgery exact sequence

$$
\cdots \rightarrow L_{m+1}\left(\mathbb{Z}\left[\pi_{1}(X)\right]\right) \stackrel{\stackrel{\partial}{\rightarrow}}{\mathcal{S}}(X) \stackrel{\eta}{\rightarrow} \mathcal{T}(X) \stackrel{\ominus}{\rightarrow} L_{m}\left(\mathbb{Z}\left[\pi_{1}(X)\right]\right)
$$

(Wall [18], Chapter 10). In general, it is possible for $\mathcal{T}(X)$ and $\mathcal{S}(X)$ to be empty: the theory involves a primary topological $K$-theory obstruction for deciding if $\mathcal{T}(X)$ is non-empty and a secondary algebraic $L$-theory obstruction for deciding if $\mathcal{S}(X)$ is non-empty. More precisely, $\mathcal{T}(X)$ is non-empty if and only if the Spivak normal fibration $\nu_{X}: X \rightarrow B G(k)\left(k\right.$ large) admits a TOP reduction $\widetilde{\nu}_{X}: X \rightarrow B \widetilde{T O P}(k)$, corresponding by the Browder-Novikov transversality construction on a degree 1 map $\rho: S^{m+k} \rightarrow T\left(\widetilde{\nu}_{X}\right)$ to a normal map

$$
(f, b)=\rho \mid: M=\rho^{-1}(X) \rightarrow X
$$


from a topological manifold. A choice of $\widetilde{\nu}_{X}$ determines a bijection

$$
\mathcal{T}(X) \cong[X, G / T O P]
$$

The algebraic surgery exact sequence of Ranicki 16]

$$
\cdots \rightarrow L_{m+1}\left(\mathbb{Z}\left[\pi_{1}(X)\right]\right) \rightarrow \mathcal{S}_{m+1}(X) \rightarrow H_{m}\left(X ; \mathbb{L}_{\bullet}\right) \stackrel{A}{\rightarrow} L_{m}\left(\mathbb{Z}\left[\pi_{1}(X)\right]\right) \rightarrow \ldots
$$

is defined for any space $X$, with $A$ the assembly map on the generalized homology group of the 1 -connective quadratic $L$-theory spectrum $\mathbb{L}_{\bullet}=\mathbb{L} \bullet\langle 1\rangle(\mathbb{Z})$ of $\mathbb{Z}$, with 0th space

$$
\mathbb{L}_{0} \simeq G / T O P
$$

and homotopy groups the simply-connected surgery obstruction groups

$$
\pi_{m}\left(\mathbb{L}_{\bullet}\right)=L_{m}(\mathbb{Z})= \begin{cases}\mathbb{Z} & \text { if } m \equiv 0(\bmod 4), \\ 0 & \text { if } m \equiv 1(\bmod 4), \\ \mathbb{Z}_{2} & \text { if } m \equiv 2(\bmod 4), \\ 0 & \text { if } m \equiv 3(\bmod 4)\end{cases}
$$

The total surgery obstruction $s(X) \in \mathcal{S}_{m}(X)$ of a simple $m$-dimensional geometric Poincaré complex is such that $s(X)=0$ if (and for $m \geq 5$ only if) $X$ is simple homotopy equivalent to an $m$-dimensional topological manifold. The surgery exact sequence of an $m$-dimensional topological manifold $M$ is isomorphic to the corresponding portion of the algebraic surgery exact sequence, with

$$
\mathcal{S}(M)=\mathcal{S}_{m+1}(M), \quad \mathcal{T}(M)=[M, G / T O P]=H_{m}\left(M ; \mathbb{L}_{\bullet}\right) .
$$

The surgery theory of topological manifolds was extended to homology manifolds in Quinn [11, 12] and Bryant, Ferry, Mio and Weinberger [1, using the 4-periodic algebraic surgery exact sequence of Ranicki [16] (Chapter 25)

$$
\cdots \rightarrow L_{m+1}\left(\mathbb{Z}\left[\pi_{1}(X)\right]\right) \rightarrow \overline{\mathcal{S}}_{m+1}(X) \rightarrow H_{m}\left(X ; \overline{\mathbb{L}}_{\bullet}\right) \stackrel{\bar{A}}{\rightarrow} L_{m}\left(\mathbb{Z}\left[\pi_{1}(X)\right]\right) \rightarrow \ldots
$$

This sequence is defined for any space $X$, with $\bar{A}$ the assembly map on the generalized homology group of the 0 -connective quadratic $L$-theory spectrum $\overline{\mathbb{L}}_{\bullet}=$ $\overline{\mathbb{L}}_{\bullet}\langle 0\rangle(\mathbb{Z})$ of $\mathbb{Z}$, with 0 th space

$$
\overline{\mathbb{L}}_{0} \simeq G / T O P \times L_{0}(\mathbb{Z}) .
$$

The 4-periodic total surgery obstruction $\bar{s}(X) \in \overline{\mathcal{S}}_{m}(X)$ of a simple $m$-dimensional geometric Poincaré complex $X$ is such that $\bar{s}(X)=0$ if (and for $m \geq 6$ only if) $X$ is simple homotopy equivalent to an $m$-dimensional homology manifold, by [1]. The homology manifold structure set $\mathcal{S}^{H}(X)$ of a simple $m$-dimensional Poincaré space $X$ is defined in the same way as $\mathcal{S}(X)$, but using homology manifolds. The surgery exact sequence of an $m$-dimensional homology manifold $M$ is isomorphic to the corresponding portion of the 4-periodic algebraic surgery exact sequence, with

$$
\mathcal{S}^{H}(M)=\overline{\mathcal{S}}_{m+1}(M) \text {. }
$$

The essential difference between surgery on homology manifolds and topological manifolds is that there is no Browder-Novikov transversality allowing the construction of normal maps from homology manifolds. Thus, the surgery exact sequence of [1] does not follow Wall [18] in relating homology manifold structures and normal invariants. Rather, the homology manifold surgery exact sequence of [1] follows the stratified surgery exact sequence of Weinberger [20] in that it relates two purely algebraically defined groups with the geometrically defined structure set. Despite 
the fact that [1] does not define homology manifold normal invariants, one can define homology manifold normal invariants $\mathcal{T}(X)$ similar to normal invariants of topological manifolds.

The homology normal invariant set $\mathcal{T}^{H}(X)$ is the bordism set of degree 1 normal maps $(f, b): M \rightarrow X$ from connected homology manifolds, with $b: \widetilde{\nu}_{M} \rightarrow \widetilde{\nu}_{X}$ a map from the canonical TOP reduction ([4]) of the Spivak normal fibration of $M$ to a $T O P$ reduction of the Spivak normal fibration of $X$. It is still the case that $\mathcal{T}^{H}(X)$ is non-empty if and only if $\nu_{X}$ is TOP reducible, but now it is necessary to also keep track of the resolution obstruction, and the homology manifolds have to be constructed using controlled topology. The following theorem allows us to use the geometric interpretation $\mathcal{T}^{H}(X)$ in the homology manifold surgery exact sequence of 1 .

Theorem 1.1 (Johnston [6]). Let $m \geq 6$. For a connected simple $m$-dimensional Poincaré space $X$ the function

$$
\begin{aligned}
& \mathcal{T}^{H}(X) \rightarrow \mathcal{T}(X) \times L_{0}(\mathbb{Z}), \\
& ((f, b): M \rightarrow X) \mapsto\left(\left(f_{T O P}, b_{T O P}\right): M_{T O P} \rightarrow M \stackrel{(f, b)}{\longrightarrow} X, i(M)\right),
\end{aligned}
$$

is a natural bijection, with $M_{T O P} \rightarrow M$ the topological degree 1 normal map determined by the canonical TOP reduction. A choice of $\widetilde{\nu}_{X}$ determines a bijection

$$
\mathcal{T}^{H}(X) \cong\left[X, G / T O P \times L_{0}(\mathbb{Z})\right] .
$$

Actually [6] (5.2) is for $m \geq 7$, but we can improve to $m \geq 6$ by a slight variation of the proof as described below.

Given the above theorem, the homology manifold surgery exact sequence of [1]

$$
\cdots \rightarrow L_{m+1}\left(\mathbb{Z}\left[\pi_{1}(X)\right]\right) \stackrel{\partial}{\rightarrow} \mathcal{S}^{H}(X) \stackrel{\eta}{\rightarrow}\left[X, G / T O P \times L_{0}(\mathbb{Z})\right] \stackrel{\ominus}{\rightarrow} L_{m}\left(\mathbb{Z}\left[\pi_{1}(X)\right]\right)
$$

becomes:

Theorem 1.2 (Bryant, Mio, Ferry and Weinberger [1]). The homology manifold structure set $\mathcal{S}^{H}(X)$ fits into the exact sequence

$$
\cdots \rightarrow L_{m+1}\left(\mathbb{Z}\left[\pi_{1}(X)\right]\right) \stackrel{\text { 品 }}{\rightarrow} \mathcal{S}^{H}(X) \stackrel{\eta}{\rightarrow} \mathcal{T}^{H}(X) \stackrel{\theta}{\rightarrow} L_{m}\left(\mathbb{Z}\left[\pi_{1}(X)\right]\right) .
$$

In particular, $\mathcal{S}^{H}(X)$ is non-empty if and only if there exists a degree 1 normal map $(f, b): M \rightarrow X$ from a homology manifold $M$ with surgery obstruction $0 \in$ $L_{m}\left(\mathbb{Z}\left[\pi_{1}(X)\right]\right)$.

If $M$ is an $m$-dimensional homology manifold, then the canonical bordism class of topological normal maps $M_{T O P} \rightarrow M$ determined by the canonical TOP reduction $\widetilde{\nu}_{M}([4])$ of the Spivak normal fibration has surgery obstruction

$$
\sigma_{*}\left(M_{T O P} \rightarrow M\right)=\bar{A}(-i(M)) \in L_{m}\left(\mathbb{Z}\left[\pi_{1}(M)\right]\right),
$$

the image (up to sign) of the resolution obstruction $i(M) \in H_{m}\left(M ; L_{0}(\mathbb{Z})\right)$ under the composite

$$
H_{m}\left(M ; L_{0}(\mathbb{Z})\right) \subseteq H_{m}\left(M ; \overline{\mathbb{L}}_{\bullet}\right) \stackrel{\bar{A}}{\rightarrow} L_{m}\left(\mathbb{Z}\left[\pi_{1}(M)\right]\right) .
$$

Remark 1.3. The algebraic surgery exact sequence has a geometric interpretation: the map $\eta: \mathcal{S}^{H}(X) \rightarrow \mathcal{T}^{H}(X)$ is given by the forgetful map and the map $\partial: L_{m+1}\left(\mathbb{Z}\left[\pi_{1}(X)\right]\right) \rightarrow \mathcal{S}^{H}(X)$ is given by "Wall realization", i.e. the homology manifold analogue of the constructions of Chapters 5 and 6 of [18] realizing the 
elements of the $L$-groups as the surgery obstructions of normal maps obtained by non-simply-connected plumbing.

To see that $\eta$ is the forgetful map, take $f: Y \rightarrow X$ and compare $\eta(f)$ with $(f: Y \rightarrow X) \in \mathcal{T}^{H}(X)$. By 1.1 above we may consider instead their images in $\mathcal{T}(X) \times L_{0}(\mathbb{Z})$. By definition of the canonical TOP-reduction of the Spivak normal fibration $\nu_{Y}$ (Ferry and Pedersen [4]), $\eta(f)$ is given by $\left(f_{T O P}: Y_{T O P} \rightarrow X, I(Y)\right)$, i.e. it agrees with the image of $f: Y \rightarrow X$ under the forgetful map.

"Wall realization" does not (yet) have an obvious geometric interpretation for homology manifolds. There are no homology manifold "handles" with nice attaching maps. Nevertheless, one can obtain the following theorem.

Theorem 1.4 (Johnston [6]). Let $m \geq 6$. For an $m$-dimensional Poincaré space $P$ with a specified homotopy equivalence $h: X \rightarrow P$, with $X$ a homology manifold, and for any element $\sigma \in L_{m+1}\left(\mathbb{Z}\left[\pi_{1}(P)\right]\right)$, the image $\partial(\sigma) \in \mathcal{S}^{H}(P)$ under the map $\partial$ : $L_{m+1}\left(\mathbb{Z}\left[\pi_{1}(P)\right]\right) \rightarrow \mathcal{S}^{H}(P)$ in the surgery exact sequence of [1] has a representative $g: Y \rightarrow P$ such that there exists a homology manifold bordism $r: W \rightarrow P \times[0,1]$ with $r \mid \partial W=g \amalg h$.

This theorem follows from [1.1 and the surgery exact sequence of [1 for $\mathcal{S}^{H}(P \times[0,1], P \times\{0,1\})$ relative to the given structures $(g, h) \in \mathcal{S}^{H}(P \times\{0,1\})$.

Theorem 1.1 is a corollary of the construction below. This construction is almost identical to that in [6], except that we have removed the use of codimension $1 \pi-\pi$ splitting to gain an extra dimension $m \geq 6$. Nonetheless, we describe the proof in detail here, because we shall need to refer to the details later as we prove a transverse variation. A transverse variation of Theorem 1.1 follows from a transverse variation of 1.5

Proposition 1.5. Let $m \geq 6$. Given a connected $m$-dimensional topological manifold $N$ and an element $I \in L_{0}(\mathbb{Z})$, there exists a degree 1 normal map $\left(f_{I}, b_{I}\right)$ : $N_{I} \rightarrow N$ from a connected homology manifold $N_{I}$ such that:

(i) The resolution obstruction of $N_{I}$ in $L_{0}(\mathbb{Z})$ is

$$
i\left(N_{I}\right)=I \in L_{0}(\mathbb{Z})
$$

(ii) The composite

$$
\left(N_{I}\right)_{T O P} \stackrel{\left(f_{T O P}, b_{T O P}\right)}{\longrightarrow} N_{I} \stackrel{\left(f_{I}, b_{I}\right)}{\longrightarrow} N
$$

is normally bordant to the identity map.

(iii) If $M$ is a connected $m$-dimensional homology manifold with resolution obstruction $i(M)=I \in L_{0}(\mathbb{Z})$, then the composite

$$
\left(M_{T O P}\right)_{I} \stackrel{\left(f_{I}, b_{I}\right)}{\longrightarrow} M_{T O P} \stackrel{\left(f_{T O P}, b_{T O P}\right)}{\longrightarrow} M
$$

is normally bordant to the identity map $M \rightarrow M$.

Proof of Theorem 1.1. Denote the given function by $\Phi: \mathcal{T}^{H}(X) \rightarrow \mathcal{T}(X) \times L_{0}(\mathbb{Z})$. Define a function

$$
\begin{aligned}
\Psi: \mathcal{T}(X) \times L_{0}(\mathbb{Z}) \rightarrow \mathcal{T}^{H}(X) \\
((f, b): N \rightarrow X, I) \mapsto\left(\left(f_{I}, b_{I}\right): N_{I} \rightarrow N \stackrel{(f, b)}{\longrightarrow} X\right),
\end{aligned}
$$

where $\left(f_{I}, b_{I}\right): N_{I} \rightarrow N$ is the result of applying Proposition 1.5 to the pair $N, I$. 


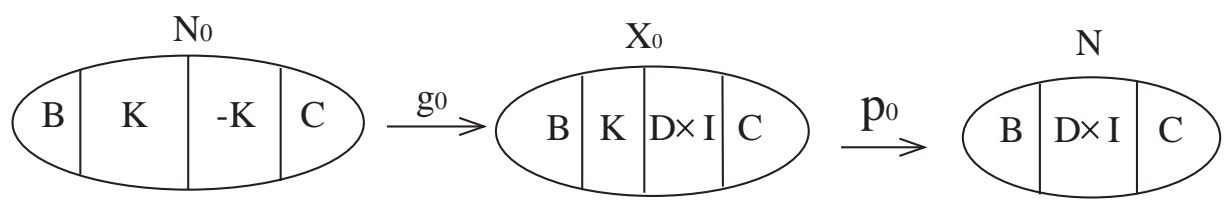

Figure 1. The construction of the spaces $N_{0}$ and $X_{0}$ and the maps $g_{0}$ and $p_{0}$.

The composition $\Phi \circ \Psi$ is the identity by 1.5 (ii), and the composition $\Psi \circ \Phi$ is the identity by 1.5 (iii).

Proof of Proposition 1.5. The construction of $\left(f_{I}, b_{I}\right): N_{I} \rightarrow N$.

The construction of $N_{I}$ is a variation on a construction found in [1] (Section 7) which is in turn a variation on the main construction of that paper. In [1] (Section 7) the construction is performed on a torus, resulting in a homology manifold not homotopy equivalent to any manifold. We perform the construction on an arbitrary topological manifold with (i), (ii) and (iii) above as the result.

Let $\sigma$ denote the element of $H_{m}\left(N ; \overline{\mathbb{L}}_{\bullet}\right)$ which corresponds to the canonical TOP reduction of the Spivak normal fibration of $N$, with the desired index $I$. Suppose we are given a sequence $\eta_{k}>0$ so that $\lim _{k \rightarrow \infty} \eta_{k}=0$.

Step I: Construct a Poincaré space $X_{0}$ with a map $p_{0}: X_{0} \rightarrow N$ so that

(i) $X_{0}$ is $\eta_{0}$-Poincaré over $N$,

(ii) $p_{0}$ is $U V^{1}$, and

(iii) $p_{0}$ has controlled surgery obstruction $\sigma \in H_{m}\left(N ; \overline{\mathbb{L}}_{\bullet}\right)$.

Slice $N$ open along the boundary of a manifold two skeleton $D$. So $N=B \cup_{D} C$. We first apply Lemma 4.4 from 1 . This will allow us to perform a small homotopy on $\operatorname{id}_{N}: N \rightarrow N$ to get a new map $q_{0}: N \rightarrow N$ which restricts to a $U V^{1}$ map on $B, C$ and $D$. Because $\left.q_{0}\right|_{D}$ is a $U V^{1}$ map, the controlled surgery obstruction group of $D \times[0,1] \stackrel{q_{0} \text { proj. }}{\longrightarrow} N$ is

$$
L^{c}\left(\begin{array}{c}
D \times[0,1] \\
\downarrow \\
N
\end{array}\right) \cong H_{m}\left(N ; \overline{\mathbb{L}}_{\bullet}\right) \cong H_{m}\left(N ; \mathbb{L}_{\bullet}\right) \oplus H_{m}\left(N ; L_{0}(\mathbb{Z})\right) .
$$

Now by Wall realization we construct a normal invariant $\sigma: K \rightarrow D \times[0,1]$ with controlled surgery obstruction $\sigma$, which is given by a controlled homotopy equivalence $k: D^{\prime} \longrightarrow D$ on one end and by the identity on the other.

Construct a Poincaré complex $X_{0}=B \cup_{i d_{D}} K \cup_{K} C$ by gluing $B$ and $C$ onto $K$. We define a map $p_{0}: X_{0} \rightarrow N$ by $\left.p_{0}\right|_{B \cup C}=i d$ and $\left.p_{0}\right|_{K}=\sigma$. By applying [1] (4.4) we may assume $p_{0}$ is $U V^{1}$. By taking sufficiently fine control we may assume that $X_{0}$ is an $\eta_{1}$-Poincaré space over $N$.

The Poincaré space $X_{0}$ has 4-periodic total surgery obstruction

$$
\bar{s}\left(X_{0}\right)=0 \in \overline{\mathcal{S}}_{m}\left(X_{0}\right),
$$

so that $X_{0}$ is homotopy equivalent to the desired homology manifold $N_{I}$ as given by [1] (6.1). In this variation of the main construction of [1 the next steps use the Poincaré space $X_{0}$ and the degree 1 normal map $g_{0}: N_{0} \rightarrow X_{0}$ representing the controlled surgery obstruction $-\sigma$ as described below. 


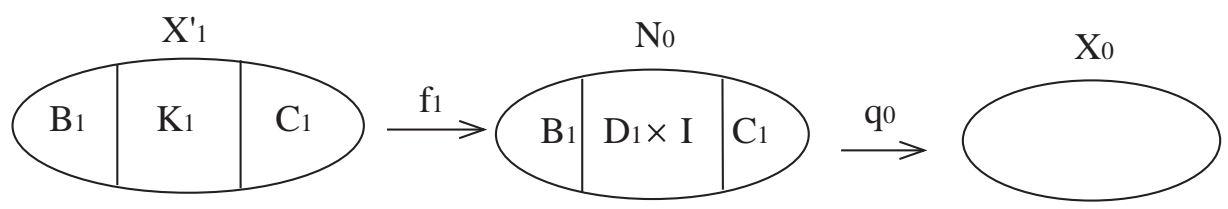

Figure 2. By construction one can surger the composition of maps $X_{1}^{\prime} \stackrel{f_{1}}{\rightarrow} N_{0} \stackrel{q_{0}}{\rightarrow} X_{0}$ to a homotopy equivalence $p_{1}: X_{1} \rightarrow X_{0}$.

Below is a brief summary of the rest of the construction in this case. It is a limiting process in which the cut and paste type construction from Step I is performed on finer and finer manifold two skeleta of manifolds $N_{k}$.

Step II: Construct a Poincaré space $X_{1}$ and a map $p_{1}: X_{1} \rightarrow X_{0}$ so that the following conditions are satisfied:

(i) The map $p_{1}$ is $U V^{1}$.

(ii) $X_{1}$ is an $\eta_{1}$-Poincaré space over $X_{0}$.

(iii) The map $p_{1}: X_{1} \rightarrow X_{0}$ is an $\epsilon_{1}$-homotopy equivalence over $N$.

(iv) For $W_{0}$ a regular neighborhood of $X_{0}$, there exist an embedding $X_{1} \rightarrow W_{0}$ and a retraction $r_{1}: W_{0} \rightarrow X_{1}$ so that $d\left(r_{0}, r_{1}\right)<\epsilon_{1}$.

Let $N_{0}$ be given by $B \cup_{D} K \cup_{D^{\prime}}-K \cup_{D} C$. Define a map

$$
g_{0}: N_{0} \rightarrow X_{0}=B \cup_{D} K \cup_{D} D \times[0,1] \cup_{D} C
$$

by the identity on $B, K$ and $C$ and by $-\sigma$ on $-K$. By [1] (4.4) we may assume that $g_{0}$ is $U V^{1}$. Let $N_{0}=B_{1} \cup_{D_{1}} C_{1}$ be a decomposition of $N_{0}$ by a finer manifold two skeleton than that of $N$. Let $q_{0}$ denote the map homotopic to $g_{0}$ which restricts to a map $U V^{1}$ on $B_{1}, C_{1}$ and $D_{1}$.

Since the map $p_{0}$ is $U V^{1}$, it induces an isomorphism

$$
\left(p_{0}\right)_{*}: H_{m}\left(X_{0} ; \overline{\mathbb{L}}_{\bullet}\right) \cong H_{m}\left(N ; \overline{\mathbb{L}}_{\bullet}\right) \text {. }
$$

Let $\sigma_{1}: K_{1} \rightarrow D_{1} \times[0,1]$ denote a Wall realization of the element of $H_{m}\left(X_{0} ; \overline{\mathbb{L}}_{\bullet}\right)$ which corresponds to $\sigma$. Define $X_{1}^{\prime}=B_{1} \cup K_{1} \cup C_{1}$ with a map $f_{1}: X_{1}^{\prime} \rightarrow N_{0}$ defined as for $p_{0}$. Consider the composition of maps $X_{1}^{\prime} \rightarrow N_{0} \rightarrow X_{0}$ and notice that it has vanishing surgery obstruction and can therefore be surgered to a small homotopy equivalence over $N$. (This type of surgery on a Poincaré space is in the tradition of Lowell Jones [7].) Denote the result of this surgery by $p_{1}: X_{1} \rightarrow X_{0}$. We may assume that $p_{1}$ is $U V^{1}$.

By choosing a sufficiently well-controlled surgery obstruction, we may assume that $X_{1}$ is $\eta_{1}$-Poincaré over $X_{0}$. By choosing $\eta_{0}$ sufficiently small we may verify conditions (iii) and (iv).

Step III: Construct a Poincaré space $X_{i+1}$ and a map $p_{i+1}: X_{i+1} \rightarrow X_{i}$ so that

(i) $p_{i+1}$ is $U V_{1}$,

(ii) $X_{i+1}$ is $\eta_{i}$-Poincaré over $X_{i}$,

(iii) $p_{i+1}$ is an $\epsilon_{i}$ equivalence over $X_{i-1}$, and

(iv) there exist an embedding $X_{i+1} \rightarrow W_{i} \subset W_{0}$ and a retraction $r_{i}: W_{0} \rightarrow X_{i+1}$ so that $d\left(r_{i}, r_{i+1}\right)<\epsilon_{i}$.

Let $g_{i}: N_{i} \rightarrow X_{i}$ be a degree 1 normal map with surgery obstruction $-\sigma \in$ $H_{m}\left(X_{i-1} ; \overline{\mathbb{L}}_{\bullet}\right) \cong H_{m}\left(N ; \overline{\mathbb{L}}_{\bullet}\right)$. By [1] (4.4) we may assume that $f_{i}$ is $U V^{1}$. Let $N_{i}=B_{i} \cup_{D_{i}} C_{i}$ be a decomposition of $N_{i}$ by a finer manifold two skeleton than 


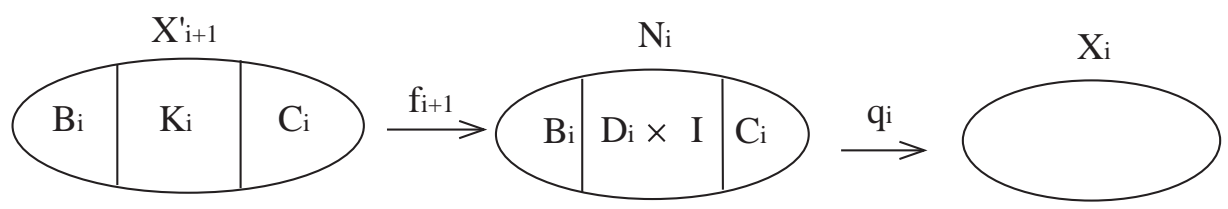

FiguRE 3. By construction one can surger the composition of maps $X_{i+1}^{\prime} \stackrel{f_{i+1}}{\rightarrow} N_{i} \stackrel{q_{i}}{\rightarrow} X_{i}$ to a homotopy equivalence $p_{i+1}: X_{i+1} \rightarrow$ $X_{i}$.

that of $N_{i-1}$. Let $q_{i}$ denote the map homotopic to $g_{i}$ which restricts to a map $U V^{1}$ on $B_{i}, C_{i}$ and $D_{i}$.

Since the map $p_{i}$ is $U V^{1}$, it induces an isomorphism

$$
H_{m}\left(X_{i} ; \overline{\mathbb{L}}_{\bullet}\right) \cong H_{m}\left(X_{i-1} ; \overline{\mathbb{L}}_{\bullet}\right) \cong H_{m}\left(N ; \overline{\mathbb{L}}_{\bullet}\right) \text {. }
$$

Let $\sigma_{i}: N_{i} \rightarrow D_{i} \times[0,1]$ denote a Wall realization of the element of $H_{m}\left(X_{i} ; \overline{\mathbb{L}}_{\bullet}\right)$ which corresponds to $\sigma$. Define $X_{i+1}^{\prime}=B_{i} \cup K_{i} \cup C_{i}$, with a map $f_{i+1}: X_{i+1}^{\prime} \rightarrow$ $N_{i}$ defined as for $p_{0}$. Consider the composition of maps $X_{i+1}^{\prime} \rightarrow N_{i} \rightarrow X_{i}$ and notice that it has vanishing surgery obstruction and can therefore be surgered to a small homotopy equivalence over $X_{i-1}$. Denote the result of this surgery by $p_{i+1}: X_{i+1} \rightarrow X_{i}$. We may assume that $p_{i+1}$ is $U V^{1}$.

By choosing a sufficiently well-controlled surgery obstruction, we may assume that $X_{i+1}$ is $\eta_{i+1}$-Poincaré over $X_{i}$. By choosing $\eta_{i-1}$ sufficiently small we may verify conditions (iii) and (iv).

Step IV: Let $N_{I}=\bigcap_{i+1}^{\infty} W_{i}$. This is the desired homology manifold. Let the map $N_{I} \rightarrow N$ be defined by $N_{I} \stackrel{\left.r\right|_{N_{I}}}{\longrightarrow} X_{0} \stackrel{p_{0}}{\rightarrow} N$.

$N_{I}$ is an $A N R$, because the limit of the retractions $r_{i}$ defines a retraction $r$ : $W_{0} \rightarrow N_{I}$. To see that $N_{I}$ is a homology manifold, we first use condition (iv) to improve the retractions $r_{i}$. Then this, together with the fact that each $X_{i}$ is an $\eta_{i}$-Poincaré space over $X_{i-1}$, can be used to show that there is a retraction $\rho: W_{0} \rightarrow N_{I}$ so that $\rho \mid: \partial W_{0} \rightarrow N_{I}$ is an approximate fibration, which shows that $N_{I}$ is a homology manifold.

This concludes the construction of $\left(f_{I}, b_{I}\right): N_{I} \rightarrow N$. It remains to show that the construction has produced the desired result.

Proof of (i). Consider the controlled surgery obstruction of $N_{k} \rightarrow X_{k} \rightarrow N_{I}$ controlled over $N_{I}$ by the identity map $N_{I} \rightarrow N_{I}$. Since the map $\rho: X_{k} \rightarrow N_{I}$ can be assumed to be $U V^{1}$, this is the same as the controlled surgery obstruction of $g_{k}: N_{k} \rightarrow X_{k}$, where $X_{k}$ has control map $\rho: X_{k} \rightarrow N_{I}$. By the argument given in [1] the control maps $\rho: X_{k} \rightarrow N_{I}$ and

$$
X_{k} \stackrel{p_{k}}{\rightarrow} X_{k-1} \stackrel{\rho}{\rightarrow} N_{I}
$$

are homotopic by a small homotopy. Thus the controlled surgery obstruction of $g_{k}$ with one control map $\rho$ is the same as that with control map $p_{k}$, but by construction this surgery obstruction was $-\sigma$, i.e., the resolution obstruction of $N_{I}$ is $I$.

Proof of (ii). Let $f_{T O P}:\left(N_{I}\right)_{T O P} \rightarrow N_{I}$ denote the degree 1 normal map induced by the TOP reduction of $N_{I}$ given by the map

$$
N_{I} \stackrel{f_{I}}{\rightarrow} N \stackrel{\nu_{N}}{\rightarrow} B T O P .
$$


By construction this map is normally bordant to $\operatorname{id}_{N}: N \rightarrow N$.

Proof of (iii). The proof is the same as injectivity of $\Phi$ in Theorem 5.2 of [6], except that we now restrict our attention to showing that the composition $\left(f_{T O P}\right)_{I} \circ f_{T O P}$ : $\left(M_{T O P}\right)_{I} \rightarrow M_{T O P} \rightarrow M$ is homology manifold normal bordant to the identity map $\operatorname{id}_{M}: M \rightarrow M$. Since any map $f_{I}: N_{I} \rightarrow N$ is an isomorphism on fundamental groups, we can avoid Lemma 5.4 of [6] and its requirement that $m \geq 7$. The map $f_{I}$ is an isomorphism on fundamental groups, because it splits as a homotopy equivalence $h: N_{I} \rightarrow X_{0}$ and the original map $p_{0}: X_{0} \rightarrow N$. The fact that $p_{0}$ is an isomorphism on fundamental groups follows from the fact that the normal invariant $\sigma: K \rightarrow D \times[0,1]$ is a Wall realization and therefore an isomorphism on fundamental groups.

A homology manifold normal bordism between $\left(f_{T O P}\right)_{I} \circ f_{T O P}:\left(M_{T O P}\right)_{I} \rightarrow$ $M_{T O P} \rightarrow M$ and $\operatorname{id}_{M}: M \rightarrow M$ is constructed as follows. First, a Poincaré bordism $k: W \rightarrow M$ is constructed between the two maps. Then, using the fact that we may assume that $\left(W,\left(M_{T O P}\right)_{I}\right)$ is a $(\pi, \pi)$ pair, we put a homology manifold structure on $W$ relative to $M$. See [6] (5.2) for details.

This completes the proof of Proposition 1.5.

For any topological block bundle $\nu: X \rightarrow \widehat{\operatorname{TOP}}(k)$, define the homology manifold bordism groups $\Omega_{m}^{H}(X, \nu)$ of normal maps $(f, b): M \rightarrow X$ from $m$-dimensional homology manifolds, with $b: \nu_{M} \rightarrow \nu$.

Corollary 1.6. For $m \geq 6$ the $m$-dimensional homology manifold bordism groups of $(X, \nu)$ are such that

$$
\Omega_{m}^{H}(X, \nu) \cong \Omega_{m}^{T O P}(X, \nu)\left[L_{0}(\mathbb{Z})\right] .
$$

Proof. Use the construction of Proposition 1.5 to define inverse isomorphisms

$$
\begin{aligned}
& \psi: \Omega_{m}^{H}(X, \nu) \rightarrow \Omega_{m}^{T O P}(X, \nu)\left[L_{0}(\mathbb{Z})\right] ; \\
&((f, b): M\rightarrow X) \mapsto\left(M_{T O P} \rightarrow M \stackrel{(f, b)}{\rightarrow} X\right)[i(M)], \\
& \psi^{-1}: \Omega_{m}^{T O P}(X, \nu)\left[L_{0}(\mathbb{Z})\right] \rightarrow \Omega_{m}^{H}(X, \nu) ; \\
&((g, c): N \rightarrow X)[I] \mapsto\left(N_{I} \rightarrow N \stackrel{(g, c)}{\rightarrow} X\right) .
\end{aligned}
$$

Thus

$$
\Omega_{m}^{H}(X, \nu)=\Omega_{m}^{T O P}(X, \nu)\left[L_{0}(\mathbb{Z})\right]=\pi_{m+k}(T(\nu))\left[L_{0}(\mathbb{Z})\right],
$$

with $T(\nu)$ the Thom space. In particular, for any space $K$ and

$$
\nu=\text { proj. : } X=K \times B T O P \rightarrow B T O P
$$

we have

$$
\Omega_{m}^{H}(K)=\Omega_{m}^{T O P}(K)\left[L_{0}(\mathbb{Z})\right]=H_{m}\left(K ; \Omega^{T O P}(\{\mathrm{pt} .\})\right)\left[L_{0}(\mathbb{Z})\right] .
$$

If $X$ is an $m$-dimensional Poincaré space with $T O P$ reducible Spivak normal fibration, then for each topological block bundle $\nu: X \rightarrow \widehat{B T O P}(k)$

$$
\Omega_{m}^{H}(X, \nu)=\pi_{m+k}(T(\nu))\left[L_{0}(\mathbb{Z})\right]=[X, G]\left[L_{0}(\mathbb{Z})\right] .
$$


For connected $X$ this is another way to see that

$$
\mathcal{T}^{H}(X)=\mathcal{T}^{T O P}(X) \times L_{0}(\mathbb{Z})=[X, G / T O P] \times L_{0}(\mathbb{Z}),
$$

as in Theorem 1.2 .

\section{Homology SUBMANifold TRANSVERSALITY UP TO BORDism}

Fix a space $P$ with a codimension $q$ bundle subspace $(Q, R, \xi)$ as in the Introduction.

We now investigate the transversality to $Q \subset P$ of a map $f: M^{m} \rightarrow P$ from an $m$-dimensional homology manifold. In the first instance, we show that if $m-q \geq 7$ then $f$ is bordant to a transverse map.

Definition 2.1. The $Q$-transverse homology manifold bordism group $\Omega_{m}^{H, Q-\pitchfork}(P)$ is the abelian group of bordism classes of maps $M^{m} \rightarrow P$ from $m$-dimensional homology manifolds which are transverse to $Q \subset P$.

There are evident forgetful maps $\Omega_{m}^{H, Q-\pitchfork}(P) \rightarrow \Omega_{m}^{H}(P)$.

Theorem 2.2. (i) The Q-transverse homology manifold bordism groups fit into an exact sequence

$$
\cdots \rightarrow \Omega_{m}^{H}(R) \rightarrow \Omega_{m}^{H, Q-\pitchfork}(P) \rightarrow \Omega_{m-q}^{H}(Q) \rightarrow \Omega_{m-1}^{H}(R) \rightarrow \ldots
$$

with

$$
\begin{aligned}
& \Omega_{m}^{H}(R) \rightarrow \Omega_{m}^{H, Q-\pitchfork}(P) ;(M, f: M \rightarrow R) \mapsto(M, M \stackrel{f}{\rightarrow} R \rightarrow P), \\
& \Omega_{m}^{H, Q-\pitchfork}(P) \rightarrow \Omega_{m-q}^{H}(Q) ;(M, g: M \rightarrow P) \mapsto\left(g^{-1}(Q), g \mid: g^{-1}(Q) \rightarrow Q\right), \\
& \Omega_{m-q}^{H}(Q) \rightarrow \Omega_{m-1}^{H}(R) ;(N, h: N \rightarrow Q) \mapsto\left(S\left(h^{*} \xi\right), S\left(h^{*} \xi\right) \rightarrow S(\xi) \rightarrow R\right) .
\end{aligned}
$$

(ii) For $m-q \geq 6$

$$
\Omega_{m}^{H, Q-\pitchfork}(P)=\Omega_{m}^{H}(P)=\Omega_{m}^{T O P}(P)\left[L_{0}(\mathbb{Z})\right] .
$$

In particular, the forgetful maps $\Omega_{m}^{H, Q-\dagger}(P) \rightarrow \Omega_{m}^{H}(P)$ are isomorphisms, and every map $M^{m} \rightarrow P$ is bordant to a $Q$-transverse map.

Proof. (i) This is a formality.

(ii) Define the $Q$-transverse topological bordism groups $\Omega_{m}^{T O P, Q-\pitchfork}(P)$ by analogy with $\Omega_{m}^{H, Q-\pitchfork}(P)$, for which there is an exact sequence

$$
\cdots \rightarrow \Omega_{m}^{T O P}(R) \rightarrow \Omega_{m}^{T O P, Q-\pitchfork}(P) \rightarrow \Omega_{m-q}^{T O P}(Q) \rightarrow \Omega_{m-1}^{T O P}(R) \rightarrow \ldots
$$

The forgetful maps $\Omega_{m}^{T O P, Q-\pitchfork}(P) \rightarrow \Omega_{m}^{T O P}(P)$ are isomorphisms, by topological transversality. Applying the 5-lemma to the map of exact sequences

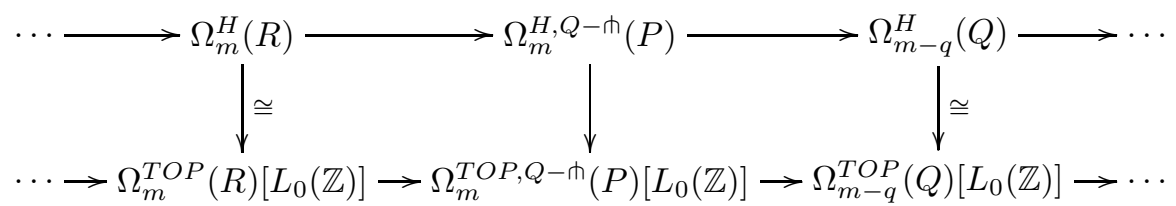

we see that the morphisms

$$
\Omega_{m}^{H, Q-\pitchfork}(P) \rightarrow \Omega_{m}^{T O P, Q-\pitchfork}(P)\left[L_{0}(\mathbb{Z})\right] ;(M, f: M \rightarrow P) \mapsto\left(M_{T O P}, f_{T O P}\right)[i(M)]
$$

are isomorphisms for $m-q \geq 6$, exactly as in the case $Q=\emptyset$. 
We wish to improve this homology manifold transversality result from "up to bordism" to "up to normal cobordism", using surgery theory.

We shall need the following variation of 1.5:

Proposition 2.3. Let $m-q \geq 7$. Given a connected $m$-dimensional topological manifold $N$, a $Q$-transverse map $g: N \rightarrow P$, and an element $I \in L_{0}(\mathbb{Z})$, there exists a degree 1 normal map $\left(f_{I}, b_{I}\right): N_{I} \rightarrow N$ from a connected homology manifold $N_{I}$ such that:

(i) The resolution obstruction of $N_{I}$ in $L_{0}(\mathbb{Z})$ is

$$
i\left(N_{I}\right)=I \in L_{0}(\mathbb{Z}) .
$$

(ii) The composite

$$
\left(N_{I}\right)_{T O P} \stackrel{\left(f_{T O P}, b_{T O P}\right)}{\longrightarrow} N_{I} \stackrel{\left(f_{I}, b_{I}\right)}{\longrightarrow} N
$$

is normally bordant to the identity map by a normal bordism. (By a Qtransverse normal bordism, since we are in the topological category.)

(iii) If $M$ is a connected $m$-dimensional homology manifold with resolution obstruction $i(M)=I \in L_{0}(\mathbb{Z})$, then the composite

$$
\left(M_{T O P}\right)_{I} \stackrel{\left(f_{I}, b_{I}\right)}{\longrightarrow} M_{T O P} \stackrel{\left(f_{T O P}, b_{T O P}\right)}{\longrightarrow} M
$$

is normally bordant to the identity map $M \rightarrow M$.

(iv) The map $\left(f_{I}, b_{I}\right)$ is $Q$-transverse.

Proof. This result follows from the following two lemmas.

Lemma 2.4. Let there be given an $m$-dimensional topological manifold $M$ for $m \geq$ 7 , such that $M$ is the union of two manifolds along a boundary component, $M=$ $M_{+} \cup_{M_{0}} M_{-}$. Then we may perform the construction of 1.5 so that the result is a homology manifold $M_{I}=\left(M_{+}\right)_{I} \cup_{\left(M_{0}\right)_{I}}\left(M_{-}\right)_{I}$ so that $M_{I}$ and $\left(M_{i}\right)_{I}$ for $i=0,+,-$ all satisfy the conclusion of 1.5 .

Lemma 2.5. Given an $(m-q)$-dimensional manifold $X$ for $m-q \geq 7$ and a $D^{q}$ bundle $\xi$ over $X$, whose total space is $E(\xi)$, we may construct a homology manifold $E_{I}$ with a map

$$
f_{I}: E_{I} \rightarrow E(\xi)
$$

which satisfies the properties of 2.3 In particular $f_{I}^{-1}(X)=X_{I}$ is a homology manifold such that $E_{I}$ is the total space of the bundle $f_{I}^{*} \xi$ and

$$
f_{I} \mid: X_{I} \rightarrow X
$$

also satisfies the properties of 1.5 .

Denote $g^{-1}(Q)$ by $N_{Q}$ and $g^{-1}(R)$ by $N_{R}$, so that $\left(N_{Q}, N_{R}, g^{*} \xi\right)$ is a codimension $q$ bundle subspace of $N$ with

$$
N=E\left(g^{*} \xi\right) \cup N_{R}
$$

By applying Lemma 2.5 to $E\left(g^{*} \xi\right)$, we get

$$
\left(f_{I}\right)_{E}: E_{I} \rightarrow E\left(g^{*} \xi\right)
$$

satisfying the above conditions. Applying 1.5 to $N_{R}$ results in

$$
\left(f_{I}\right)_{R}: R_{I} \rightarrow N_{R}
$$




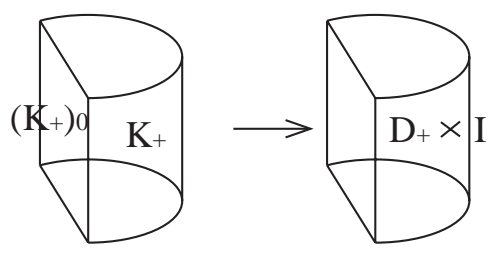

FiguRE 4. The Wall realization $K_{+}$.

By Lemma 2.4 we may apply the construction to $N_{R}$ and $E\left(g^{*} \xi\right)$ simultaneously so that the resulting homology manifolds $R_{I}$ and $E_{I}$ and maps agree on their boundaries. This proves Proposition 2.3.

Proof of Lemma 2.4 Take a manifold two-skeleton of $M$ which is the union of manifold two skeletons for $M_{+}$and $M_{-}$along a manifold two skeleton for $M_{0}$. Denote this two skeleton and its boundary by

$$
B=B_{+} \cup_{B_{0}} B_{-} \text {and } D=D_{+} \cup_{D_{0}} D_{-} .
$$

We shall perform the construction of 1.5 simultaneously on $M_{+}$and $M_{-}$. What was one controlled surgery obstruction in the original construction

$$
\sigma=(0, I) \in H_{m}\left(M ; \overline{\mathbb{L}}_{\bullet}\right)
$$

is now two controlled surgery obstructions

$$
\sigma_{+}=(0, I) \in H_{m}\left(M_{+} ; \overline{\mathbb{L}}_{\bullet}\right), \quad \sigma_{-}=(0, I) \in H_{m}\left(M_{-} ; \overline{\mathbb{L}}_{\bullet}\right) .
$$

In Step I, we apply 1] (4.4) to get a map $q_{0}: M \rightarrow M$ which restricts to $U V^{1}$ maps $D_{i} \rightarrow M_{i}, i=0,+,-$, and is itself $U V^{1}$. Thus the controlled surgery obstruction groups are given by

$$
L^{c}\left(\begin{array}{c}
D_{i} \times[0,1] \\
\downarrow \\
M_{ \pm}
\end{array}\right) \cong H_{m}\left(M_{ \pm} ; \overline{\mathbb{L}}_{\bullet}\right) \cong H_{m}\left(M_{ \pm} ; \mathbb{L}_{\bullet}\right) \oplus H_{m}\left(M_{ \pm} ; L_{0}(\mathbb{Z})\right)
$$

Since $M_{ \pm}$are manifolds with boundary, $m \geq 7$ is required. Take a Wall realization $K_{ \pm}$of $(0, I)$. Glue this into $M_{ \pm}$to create the first Poincaré space of the construction, which comes with a map $p_{0}: X_{0} \rightarrow M$ and a Poincaré decomposition $X_{0}=$ $\left(X_{0}\right)_{+} \cup\left(X_{0}\right)_{-}$which is respected by $p_{0}$.

We must exercise some care to get the Wall realizations to agree on their boundaries.

Denote $\partial K_{ \pm}=\left(K_{ \pm}\right)_{0}$. The images of $\left(K_{ \pm}\right)_{0} \rightarrow D_{0} \times[0,1]$ agree in

$$
L^{c}\left(\begin{array}{c}
D_{0} \times[0,1] \\
\downarrow \\
M_{0}
\end{array}\right) \cong H_{m-1}\left(M_{0} ; \overline{\mathbb{L}}_{\bullet}\right) .
$$

Each is again $(0, I)$, the image of $(0, I)$ under the boundary maps

$$
H_{m}\left(M_{ \pm} ; \overline{\mathbb{L}}_{\bullet}\right) \rightarrow H_{m-1}\left(M_{0} ; \overline{\mathbb{L}}_{\bullet}\right) .
$$

Since the surgery obstructions of $\partial K_{+}=\left(K_{+}\right)_{0}$ and $\partial K_{-}=\left(K_{-}\right)_{0}$ agree, we may glue them together along their common boundary $D_{0}$ to get a normal invariant with vanishing controlled surgery obstruction, i.e., we may surger the map

$$
\partial K_{+} \cup \partial K_{-} \rightarrow D_{0} \times[0,1]
$$




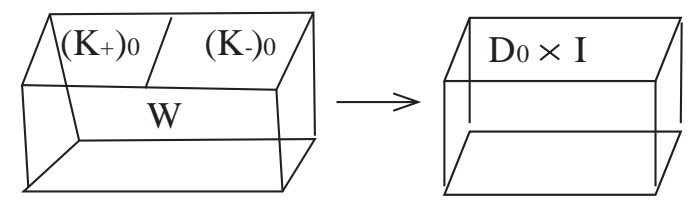

Figure 5 . The trace of surgery on $\left(K_{+}\right)_{0} \cup\left(K_{-}\right)_{0} \rightarrow D_{0} \times[0,1]$ can be used to "match up" the boundaries of the Wall realizations.

to a controlled homotopy equivalence. Let $W$ denote the trace of this surgery. Denote $K_{-}^{\prime}=K_{-} \cup_{\left(K_{-}\right)_{0}} W$, and extend the map $K_{-} \rightarrow D_{-} \times[0,1]$ in the obvious way. It is a Wall realization of $(0, I)$, which agrees with $K_{+}$on its boundary.

If we perform the construction with two Wall realizations which agree on their boundaries, then we may glue them together to get $X_{0}=\left(X_{0}\right)_{+} \cup\left(X_{0}\right)_{-}$. Furthermore, since the boundary of the Wall realizations is itself a Wall realization, the intersection

$$
\left(X_{0}\right)_{+} \cap\left(X_{0}\right)_{-}
$$

is itself the first stage of the given construction on $M_{0}$. Similarly the union $X_{0}$, which is the result of gluing in the union of the Wall realizations, is the first stage of the construction on $M$.

To preserve this decomposition throughout the construction requires only that we repeat the above type of construction in later steps.

Proof of Lemma 2.5. Let $B_{X}$ denote a manifold two skeleton of $X$. Since $E(\xi)$ and $X$ are of the same homotopy type, we could easily construct a manifold two skeleton of $E(\xi)$ by thickening a manifold two skeleton of $X$, i.e., $E\left(c^{*} \xi\right)$, where $c: B_{X} \rightarrow X$. Recall that the construction requires a fine manifold two skeleton. Let $E_{X}$ denote the manifold two skeleton of an $\epsilon$-neighborhood of $X$ in $E(\xi)$. We may extend this to a fine manifold two skeleton of $E(\xi)$ by

$$
B=E_{X} \cup B_{S},
$$

where $B_{S}$ is a manifold two skeleton of the complement of the given $\epsilon$-neighborhood of $X$.

Here $E_{X} \cap B_{S}$ is the manifold two skeleton of $S(\xi)$ for the given $\epsilon$-sphere bundle. We shall now perform the construction of 2.4 on this decomposition of $E(\xi)$, but with the added requirement that the result of the construction on the small neighborhood of $X$ is itself a bundle over the desired $X_{I}$. This is done by preserving the bundle throughout the construction. Take a Wall realization of

$$
(0, I) \in H_{m-q}\left(X ; \overline{\mathbb{L}}_{\bullet}\right)
$$

denoted by

$$
\sigma_{X}: N_{X} \rightarrow D_{X} \times[0,1] .
$$

Let $\sigma_{E}$ denote the bundle map on the pullback

$$
N_{E}=\sigma_{X}^{*} \xi^{\prime}
$$

where $\xi^{\prime}$ is the bundle induced on $D_{X} \times[0,1]$ by $\xi$. The result is

$$
\sigma_{E}: N_{E} \rightarrow E\left(\xi \mid D_{X} \times[0,1]\right),
$$


the image of $\sigma_{X}$ under the transfer map. The corresponding surgery obstruction is thus the image of $(0, I)$ under the transfer map

$$
H_{m-q}\left(X ; \overline{\mathbb{L}}_{\bullet}\right) \rightarrow H_{m}\left(E(\xi) ; \overline{\mathbb{L}}_{\bullet}\right) .
$$

One need only check that this map takes $(0, I)$ to $(0, I)$ to see that we may preserve the bundle throughout the construction.

Given a simple $m$-dimensional Poincaré space $X$ with a map $g: X \rightarrow P$ to a space $P$ with a codimension $q$ bundle subspace $Q \subset P$, let $\mathcal{T}^{H, Q-\pitchfork}(X)$ be the set of bordism classes of normal maps $(f, b): M \rightarrow X$ from $m$-dimensional homology manifolds such that $g f: M \rightarrow P$ is transverse to $Q \subset P$.

Theorem 2.6. Given $X, g: X \rightarrow P$ and $Q \subset P$ as above such that $m-q \geq 7$.

(i) The forgetful function $\mathcal{T}^{H, Q-\pitchfork}(X) \rightarrow \mathcal{T}^{H}(X)$ is a bijection.

(ii) Every map $f: M \rightarrow P$ from an m-dimensional homology manifold is bordant to a map transverse to $Q \subset P$.

Proof. (i) The functions

$$
\begin{aligned}
& \mathcal{T}^{H}(X) \rightarrow \mathcal{T}^{T O P}(X) \times L_{0}(\mathbb{Z}) ;((f, b): M \rightarrow X) \mapsto\left(\left(f_{T O P}, b_{T O P}\right), i(M)\right), \\
& \mathcal{T}^{H, Q-\pitchfork}(X) \rightarrow \mathcal{T}^{T O P, Q-\pitchfork}(X) \times L_{0}(\mathbb{Z}) ;((f, b): M \rightarrow X) \mapsto\left(\left(f_{T O P}, b_{T O P}\right), i(M)\right)
\end{aligned}
$$

are bijections by Theorem 1.1 and its $Q$-transverse variation 2.3 The forgetful function

$$
\mathcal{T}^{T O P, Q-\pitchfork}(X) \rightarrow \mathcal{T}^{T O P}(X)
$$

is a bijection by topological transversality, so that

$$
\mathcal{T}^{H, Q-\pitchfork}(X)=\mathcal{T}^{T O P, Q-\pitchfork}(X) \times L_{0}(\mathbb{Z})=\mathcal{T}^{T O P}(X) \times L_{0}(\mathbb{Z})=\mathcal{T}^{H}(X) .
$$

(ii) Unfortunately (i) does not apply to an arbitrary map. We may get around this by factoring any map $f: M \rightarrow P$ through a homotopy equivalence $\widehat{f}: M \rightarrow \bar{P}$. Any map $f$ is homotopic to $\widehat{f} \circ \bar{f}$ such that $\widehat{f}: \bar{P} \rightarrow P$ is a Serre fibration and $\bar{f}$ is a homotopy equivalence. Now by (i), $\bar{f}$ is normally cobordant to a transverse map, and hence $f$ is bordant to a transverse map.

Given a simple $m$-dimensional Poincaré duality space $X$ with a map $g: X \rightarrow P$, as above, we may also study the $Q$-transverse homology manifold structure set. Denoted by $\mathcal{S}^{H, Q-\pitchfork}(X)$, this is the set of equivalence classes of pairs $(M, h)$ with $M$ an $m$-dimensional homology manifold and $h: M \rightarrow X$ a simple homotopy equivalence such that $g h: M \rightarrow P$ is transverse to $Q \subset P$, with $\left(M_{1}, h_{1}\right) \simeq\left(M_{2}, h_{2}\right)$ if there exists an $s$-cobordism $\left(W ; M_{1}, M_{2}\right)$ with a simple homotopy equivalence of the type

$$
\left(f ; h_{1}, h_{2}\right):\left(W ; M_{1}, M_{2}\right) \rightarrow X \times([0,1] ;\{0\},\{1\})
$$

such that the composite

$$
W \stackrel{f}{\rightarrow} X \times[0,1] \stackrel{\text { proj. }}{\rightarrow} X \stackrel{g}{\rightarrow} P
$$

is transverse to $Q \subset P$.

Remark 2.7. The isomorphism

$$
\mathcal{S}^{H, Q-\pitchfork}(X) \cong \mathcal{S}^{H}(X)
$$

will follow from $s$-transversality for homology submanifolds, Theorem 4.1 just as Theorem 2.6] followed from 2.3 
Before dealing with transversality up to $s$-cobordism, we turn our attention to some splitting theorems. These theorems follow directly from transversality up to normal bordism and are useful in the proof of transversality up to $s$-cobordism.

\section{Codimension $q$ Splitting of homology manifolds}

Fix a space $P$ with a codimension $q$ bundle subspace $(Q, R, \xi)$, as in Section 2 .

Wall [18] (Chapter 11) defined the codimension $q$ splitting obstruction groups $L S_{*}(\Phi)$ to fit into an exact sequence

$$
\begin{aligned}
\ldots & \rightarrow L_{m+1}\left(\mathbb{Z}\left[\pi_{1}(R)\right] \rightarrow \mathbb{Z}\left[\pi_{1}(P)\right]\right) \rightarrow L S_{m-q}(\Phi) \\
& \rightarrow L_{m-q}\left(\mathbb{Z}\left[\pi_{1}(Q)\right]\right) \stackrel{\xi^{!}}{\rightarrow} L_{m}\left(\mathbb{Z}\left[\pi_{1}(R)\right] \rightarrow \mathbb{Z}\left[\pi_{1}(P)\right]\right) \rightarrow \ldots
\end{aligned}
$$

with $\xi^{!}$the transfer maps induced by $\xi$.

From now on, we assume that $P$ is an $m$-dimensional Poincaré space and that $Q \subset P$ is an $(m-q)$-dimensional Poincaré subspace, with $(R, S(\xi))$ an $m$-dimensional Poincaré pair.

Definition 3.1. (i) A simple homotopy equivalence $f: M \rightarrow P$ from an $m$ dimensional homology manifold splits along $Q \subset P$ if $f$ is $s$-cobordant to a simple homotopy equivalence (also denoted by $f$ ) which is transverse to $Q \subset P$,

$$
f=E(g) \cup_{S(g)} h: M=E\left(g^{*} \xi\right) \cup_{S\left(g^{*} \xi\right)} Z \rightarrow P=E(\xi) \cup_{S(\xi)} R,
$$

and such that the restrictions

$$
g=f\left|: N=f^{-1}(Q) \rightarrow Q, \quad h=f\right|: Z=f^{-1}(R) \rightarrow R
$$

are simple homotopy equivalences.

(ii) The split structure set $\mathcal{S}^{H}(P, Q, \xi)$ is the set of homology manifold structures on $P$ which split along $Q \subset P$.

For any simple homotopy equivalence $f: M \rightarrow P$ from an $m$-dimensional topological manifold $M$ there is defined a codimension $q$ splitting obstruction

$$
s_{Q}(f) \in L S_{m-q}(\Phi)
$$

such that $s_{Q}(f)=0$ if (and for $m-q \geq 5$ only if) $f$ splits in the topological manifold category. The image of $s_{Q}(f)$ in $L_{m-q}\left(\mathbb{Z}\left[\pi_{1}(Q)\right]\right)$ is the surgery obstruction $\sigma_{*}(g)$ of the normal map $g=f \mid: N=f^{-1}(Q) \rightarrow Q$ obtained by topological transversality. See Wall [18] (Chapter 11) and Ranicki 15] (pp. 572-577) for details.

Theorem 3.2. Let $m-q \geq 7$.

(i) A simple homotopy equivalence $f: M \rightarrow P$ from an $m$-dimensional homology manifold with a codimension $q$ bundle subspace $(Q, R, \xi)$ splits along $Q \subset P$ if and only if an obstruction $s_{Q}(f) \in L S_{m-q}(\Phi)$ vanishes.

(ii) The split homology manifold structure set $\mathcal{S}^{H}(P, Q, \xi)$ fits into an exact sequence

$$
\cdots \rightarrow L S_{m-q+1}(\Phi) \rightarrow \mathcal{S}^{H}(P, Q, \xi) \rightarrow \mathcal{S}^{H}(P) \rightarrow L S_{m-q}(\Phi) .
$$

Proof. Define $L S_{m-q}^{H}(\Phi)$ to be the group of obstructions in the exact sequence

$$
\mathcal{S}^{H}(P, Q, \xi) \rightarrow N I^{H, Q-\dagger}(X) \rightarrow L S_{m-q}^{H}(\Phi) .
$$

Consider the homology manifold normal invariant given by $f: M \rightarrow P$; by [2.6 $f$ is normally bordant, say via $W \rightarrow P \times I$, to a map $g: M^{\prime} \rightarrow P$ such that $g$ is 


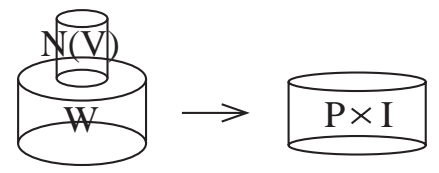

Figure 6. A homology manifold normal invariant of $(P \times$ $[0,1], R \times 0)$.

transverse to $Q$. In particular, $g^{-1}(Q)=Y$ is a homology manifold with a normal neighborhood $N(Y)=g^{*}(\xi)$.

Thus, this transverse normal invariant defines a splitting obstruction which lives in $L S_{m-q}^{H}(\Phi)$. Since we do not a priori have an understanding of $L S_{m-q}^{H}(\Phi)$, we must study it by comparing to the obstruction groups $L_{m+1}\left(\pi_{1}(R) \rightarrow \pi_{1}(P)\right)$, $L_{m-q}(Q)$ and the surgery exact sequences for $\mathcal{S}^{H}(P \times[0,1], R \times 0)$ and $\mathcal{S}^{H}(Q)$.

There is clearly a commutative diagram with vertical maps given by restriction as follows:

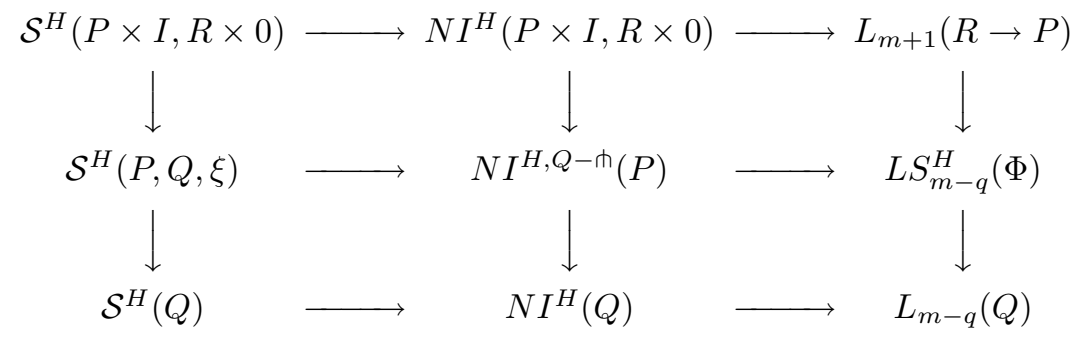

The splitting obstruction $\sigma(f) \in L S_{m-q}^{H}(\Phi)$ can be understood as a two stage obstruction as follows. First the normal invariant $g \mid: Y \rightarrow Q$ defines an obstruction $\sigma_{Q}(f) \in L_{m-q}(Q)$. If this obstruction vanishes, then, by the surgery exact sequence of [1], $g \mid: Y \rightarrow Q$ is normally cobordant to a simple homotopy equivalence. Let $V \rightarrow Q \times[0,1]$ denote this normal cobordism, and let $N(V) \rightarrow E(\xi) \times[0,1]$ denote the corresponding pullback of $\xi$. We can define a homology manifold normal invariant of $P \times[0,1] \mathrm{rel} P \times 0, E(\xi) \times 1$ by gluing $N(V)$ to $W$. There is now defined an obstruction $\sigma_{R}(f) \in L_{m+1}\left(\pi_{1}(R) \rightarrow \pi_{1}(P)\right)$ to this normal invariant being equivalent to a simple homotopy equivalence of $P \times[0,1]$, i.e. an s-cobordism from $f: M \rightarrow P$ to some map $h: N \rightarrow P$.

If $\sigma(f) \in L S_{m-q}(\Phi)$ vanishes, then both $\sigma_{Q}(f)$ and $\sigma_{R}(f)$ are defined and vanish, so that $f$ is $s$-cobordant to a split map. Conversely, if $f$ is $s$-cobordant to a split map, then both $\sigma_{Q}(f)$ and $\sigma_{R}(f)$ are defined and vanish.

Example 3.3. (i) If $q=1, \xi$ is trivial, and $R=R_{1} \sqcup R_{2}$ is disconnected with $\pi_{1}(Q) \cong \pi_{1}\left(R_{1}\right)$, then $L S_{*}(\Phi)=0$, there is no obstruction in Theorem 3.2 and

$$
\mathcal{S}^{H}(P)=\mathcal{S}^{H}(P, Q, \xi) .
$$

This is the homology manifold $\pi-\pi$ codimension 1 splitting theorem already obtained by Johnston 6 .

(ii) If $q \geq 3$, the codimension $q$ splitting obstruction is just the ordinary surgery obstruction

$$
s_{Q}(f)=\sigma_{*}(g) \in L S_{m-q}(\Phi)=L_{m-q}\left(\mathbb{Z}\left[\pi_{1}(Q)\right]\right)
$$


of the restriction $g=f \mid: f^{-1}(Q) \rightarrow Q$, with an exact sequence

$$
\cdots \rightarrow L_{m-q+1}\left(\mathbb{Z}\left[\pi_{1}(Q)\right]\right) \rightarrow \mathcal{S}^{H}(P, Q, \xi) \rightarrow \mathcal{S}^{H}(P) \rightarrow L_{m-q}\left(\mathbb{Z}\left[\pi_{1}(Q)\right]\right) .
$$

This is the homology manifold Browder splitting theorem already obtained by Johnston [6].

\section{Homology SUBMANIFOLD TRANSVERSALITY UP TO $s$-COBORDISM}

We proceed to prove homology manifold transversality up to $s$-cobordism, using the above results.

Theorem 4.1. Let $f: M \rightarrow P=E(\xi) \cup_{S(\xi)} R$ be a map from an m-dimensional homology manifold $M$ to a space $P$ with a codimension $q$ bundle subspace $(Q, R, \xi)$. If $m-q \geq 7$, then $f$ is s-transverse to $Q \subset P$.

Proof. For $Q \subset P$ of codimension $q \geq 3$ this was proved in [6].

We prove the theorem here for codimension $q=1,2$. First we may assume that the map $f: M \rightarrow P$ is a homotopy equivalence by factoring the original map through a Serre fibration. This results in

$$
M \stackrel{\widehat{f}}{\longrightarrow} \bar{P} \stackrel{\bar{f}}{\longrightarrow} P,
$$

homotopic to the original $f$, so that $\widehat{f}$ is a homotopy equivalence, $\bar{f}$ is a Serre fibration, and $\bar{P}$ has a codimension $q$ subset $\bar{Q}$ so that the normal bundle of $\bar{Q}$ in $\bar{P}$ is the pullback of the normal bundle of $Q$ in $P$. To achieve $Q$-transversality for $f$, clearly it would suffice to achieve $\bar{Q}$ transversality for $\widehat{f}$. Thus we may assume that $f: M \rightarrow P$ is a homotopy equivalence. By Theorem 2.6 (ii) we have that $f$ is bordant to a $Q$-transverse map. From here the proof proceeds in two steps, given by the following lemma.

Lemma 4.2. If $f: M \rightarrow P$ is a homotopy equivalence as above and

$$
F: W \rightarrow P \times[0,1]
$$

is a homology manifold bordism from $f$ to $g: M^{\prime} \rightarrow P$, then:

(i) The map $f$ is homotopy equivalent to a map $\bar{g}: M \rightarrow P$, which factors through a homotopy equivalence to a Poincaré space $X, h: M \rightarrow X$ with $g^{\prime \prime}: X \rightarrow P$ such that $\bar{g}=g^{\prime \prime} \circ h$, and $g^{\prime \prime}$ is Poincaré transverse to $Q$, i.e. the inverse image of $Q$ is a Poincaré space with normal bundle the pullback of the normal bundle of $Q$.

(ii) The map $\bar{g}$ is s-cobordant to a $Q$-transverse map.

Proof of Lemma 4.2. (i) The key idea of this proof is to use a patch space structure on $W$ to achieve the desired result. Because $W$ is a homology manifold, it has a patch space structure with only two patches. Let

$$
H: W^{\prime} \rightarrow W
$$

be such a structure, where

$$
W^{\prime}=W_{+} \cup_{W_{0}} W_{-}
$$

gives $W$ as a union of manifolds glued along a homotopy equivalence

$$
h_{W}: W_{0} \rightarrow W_{0} \text {. }
$$


Furthermore, we may assume that

$$
\partial W^{\prime}=X \amalg X^{\prime}
$$

is such that

$$
H \mid: \partial W^{\prime} \rightarrow M \amalg M^{\prime}
$$

restricts to two patch space structures

$$
X=X_{+} \cup X_{-}
$$

and

$$
X^{\prime}=X_{+}^{\prime} \cup X_{-}^{\prime}
$$

with gluing maps

$$
h_{X}: X_{0} \rightarrow X_{0}
$$

and

$$
h_{X^{\prime}}: X_{0}^{\prime} \rightarrow X_{0}^{\prime}
$$

on each of $M$ and $M^{\prime}$.

We construct a patch space structure for $M^{\prime}$ as follows: First construct a patch space structure

$$
H \mid: Q^{\prime \prime} \rightarrow Q^{\prime}
$$

for the homology manifold $Q^{\prime}=g^{-1}(Q)$. Thicken this patch space structure to a patch space structure for $\nu\left(Q^{\prime}\right)$, the pullback of the normal bundle of $Q$. Then construct a patch space structure for

$$
M^{\prime} \backslash \nu\left(Q^{\prime}\right)
$$

relative to the structure for $\partial \nu\left(Q^{\prime}\right)$. Finally construct a patch space structure for $W$ relative to this structure for $M^{\prime}$. In this manner a patch space structure for $W$ is constructed so that the map

$$
g \circ H \mid: X^{\prime} \rightarrow P \times\{1\}
$$

is Poincaré transverse to $Q$.

Consider the map

$$
G=F \circ H: W^{\prime} \rightarrow W \rightarrow P \times[0,1] .
$$

We shall show that $G$ is homotopic rel $X^{\prime}$ to a map which is Poincaré transverse to $Q$. First consider $G \mid W_{+}$. We may use manifold transversality to make this map transverse to

$$
Q \times[0,1] \subset P \times[0,1],
$$

which inherits a codimension- $q$ structure from $Q \subset P$. If the homotopy equivalence

$$
h_{W}: W_{0} \rightarrow W_{0}
$$

splits along

$$
Q_{0}=\left(\left.G\right|_{W+}\right)^{-1}(Q \times[0,1]) \cap W_{0},
$$

then $G \mid W_{-}$is $Q \times[0,1]$-transverse along its boundary $W_{0}$, and we may use manifold transversality to homotope $G \mid W_{-}$rel $W_{0}$ to a $Q$-transverse map. Unfortunately there is no guarantee that the homotopy equivalence $h_{W}$ splits along $Q_{0}$. There 


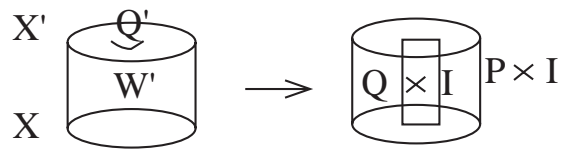

Figure 7. We would like to extend Poincaré transversality for the map $G$ from $X^{\prime}$ to all of $W^{\prime}$.

is a priori a splitting obstruction $\sigma \in L S_{m-q}(\Phi)$, where $\Phi$ is defined by the pair $Q_{0} \subset W_{0}$.

We shall show that by changing to a different patch space structure $\bar{H}: \bar{W} \rightarrow$ $W$, we may assume that this obstruction vanishes. First we need to change the patch space structure slightly so that $L S_{m-q}(\Phi)$ acts on the set of possible patch space structures. Our action will be by $L S_{m-q}(\Psi)$, where $\Psi$ is defined by the pair $Q^{\prime \prime} \subset X^{\prime}$.

The first step is to show that we may assume

$$
L S_{m-q}(\Phi) \cong L S_{m-q}(\Psi) .
$$

This is achieved by performing Poincaré surgery to fix the fundamental groups of $Q_{0}$ and $W_{0}$; but these are the boundaries of the manifold two skeletons of

$$
Q^{\prime}=\left(\left.G\right|_{W_{+}}\right)^{-1}(Q \times[0,1])
$$

and $W^{\prime}$. Therefore the fundamental groups of $Q^{\prime} \subset W^{\prime}$ agree with $\Phi$, and we may view the problem of changing $\Phi$ to agree with $\Psi$ as a problem about $Q^{\prime} \subset W^{\prime}$. This is just fundamental group Poincaré surgery on

$$
G: W^{\prime} \rightarrow P \times[0,1],
$$

or rather manifold surgery on the manifold two skeleton of $W^{\prime}$,

$$
G \mid: W_{+} \rightarrow W_{+} \text {. }
$$

Now we can assume that both $Q^{\prime \prime} \subset X^{\prime}$ and $Q_{0} \subset W_{0}$ define the same group $L S_{m-q}(\Phi)$. This group acts on the patch space structures of $X^{\prime}$ by acting on $h_{X^{\prime}}$ as follows: $L_{m-q}\left(Q^{\prime \prime}\right)$ acts on the patch space structures

$$
h \mid: Q^{\prime \prime} \rightarrow Q^{\prime}
$$

for $Q^{\prime \prime}=Q_{+}^{\prime \prime} \cup Q_{-}^{\prime \prime}$ by acting on the gluing homotopy equivalence

$$
h_{Q^{\prime \prime}}: Q_{0}^{\prime \prime} \rightarrow Q_{0}^{\prime \prime}
$$

Given an element $\sigma \in L_{m-q}\left(Q^{\prime \prime}\right)$, denote its Wall realization by $\sigma: K \rightarrow Q_{0}^{\prime \prime} \times[0,1]$, where $\partial K=Q_{0}^{\prime \prime} \amalg \widehat{Q}_{0}$. The new patch space structure is given by $\widehat{Q^{\prime \prime}}=\widehat{Q_{+}^{\prime \prime}} \cup Q_{-}^{\prime \prime}$, where $\widehat{Q_{+}^{\prime \prime}}=Q_{+}^{\prime \prime} \cup_{i d_{Q_{0}^{\prime \prime}}} K$. The new gluing map is now $h_{\widehat{Q^{\prime \prime}}}=\sigma \mid \widehat{Q^{\prime \prime}}$. A Poincaré bordism from $Q^{\prime \prime}$ to $\widehat{Q^{\prime \prime}}$ is given by $\widehat{Q_{+}^{\prime \prime}} \times[0,1] \cup_{\sigma} Q_{-}^{\prime \prime} \times[0,1]$. where $\sigma$ is viewed as a map $\sigma: K \times 0 \rightarrow Q_{0}^{\prime \prime} \times[0,1]$. The group $L_{m}\left(X^{\prime} \backslash Q^{\prime \prime}\right)$ acts similarly on the structure

$$
h_{X} \mid: X_{0}^{\prime} \backslash Q_{0}^{\prime \prime} \rightarrow X_{0}^{\prime} \backslash Q_{0}^{\prime \prime} .
$$

The fact that we really had an element of $L S_{m-q}(\Phi)$ insures that the Wall realizations will glue together to a composite which we denote by $K_{\sigma}$, and that the patch spaces will glue together to give a new patch space structure, $X^{\prime \prime}$. 


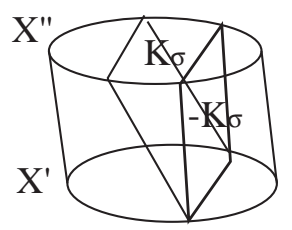

Figure 8 . The action of $\sigma$ on $X^{\prime}$ by $K_{\sigma}$ produces a new space $X^{\prime \prime}$ and adds the above to $W^{\prime}$. In particular, it adds $-K_{\sigma}$ to $W_{0}$.

Claim. The action of

$$
\sigma \in L S_{m-q}(\Psi)
$$

results in a new patch space structure whose transversality obstruction differs by $-\sigma$ from the previous one, i.e., with the correct choice of $\sigma$ the transversality obstruction vanishes.

Proof of claim. If we consider the addition of $K_{\sigma} \times[0,1]$ as in the diagram, it becomes clear that we have changed $W_{0}$ by $-K_{\sigma}$, as asserted.

The map $G$ is now homotopic to a Poincaré transverse map, and $\left.G\right|_{X}$ is the desired map $g^{\prime \prime}$ Poincaré transverse to $Q$. The original map $f$ is homotopic to $g^{\prime \prime} \circ h$ where $h: M \rightarrow X$ is the homotopy inverse of $H \mid: X \rightarrow M$. This completes the proof of Lemma 4.2 (i).

(ii) Suppose we are given

$$
\bar{g}=g^{\prime \prime} \circ h: M \rightarrow P,
$$

for the homotopy equivalence $h: M \rightarrow X$ and the Poincare transverse map $g^{\prime \prime}$ : $X \rightarrow P$. Let $Q^{\prime}$ denote $\left(g^{\prime \prime}\right)^{-1}(Q)$, so that by assumption $Q^{\prime}$ is a Poincaré space. If $h$ is $s$-cobordant to a homotopy equivalence $\widehat{h}: \widehat{M} \rightarrow X$ which is split over $Q^{\prime}$, then $g^{\prime \prime} \circ \widehat{h}$ is homology manifold transverse to $Q$. There is an obstruction $\sigma$ to splitting $h$ which lives in $L S_{m-q}(\Phi)$, where $\Phi$ is defined by the pair $Q^{\prime} \subset X$. Let $\sigma$ denote this obstruction.

Because $Q^{\prime}$ was constructed by the method of $4.2(\mathrm{i})$, it has a two-patch structure

$$
Q^{\prime}=Q_{+}^{\prime} \cup Q_{-}^{\prime} \text { and } Q_{0}^{\prime}=Q_{+}^{\prime} \cap Q_{-}^{\prime}
$$

which agrees with the two-patch space structure of $X$, i.e. $Q_{+}^{\prime} \subset X_{+}, Q_{-}^{\prime} \subset X_{-}$ and $Q_{0}^{\prime} \subset X_{0}$. If $\Psi$ is defined by the pair $Q_{0}^{\prime} \subset X_{0}$, there is a natural isomorphism

$$
L S_{m-q}(\Psi) \cong L S_{m-q}(\Phi) \text {. }
$$

$L S_{m-q}(\Psi)$ acts on the possible patch space structures $h: M \rightarrow X$ on $M$ as follows. Recall that, by construction, the gluing map

$$
h_{X}: X_{0} \rightarrow X_{0}
$$

splits along $Q_{0}^{\prime} \subset X_{0}$. We can change the patch space structure on $X$, if we make sure that it has a map to the original $X$ which is Poincaré transverse to $Q^{\prime}$. In particular, the new map

$$
h_{Y}: Y_{0} \rightarrow Y_{0}
$$

must split along the inverse image of $Q^{\prime}$. Take $h_{X}$ and act on it by $L S_{m-q}(\Phi)$, by acting on

$$
h_{Q^{\prime}} \mid: Q_{0}^{\prime} \rightarrow Q_{0}^{\prime}
$$


by $L_{m-q}\left(Q_{0}^{\prime}\right)$ and by acting on

$$
h_{X} \mid: X_{0} \backslash Q_{0}^{\prime} \rightarrow X_{0} \backslash Q_{0}^{\prime}
$$

by $L_{m}\left(X_{0} \backslash Q_{0}^{\prime}\right)$. The result fits together along $\partial \nu\left(Q^{\prime}\right)$ by definition of $L S_{m-q}(\Psi)$. By construction, there is a map $k: Y \rightarrow X$ which is a homotopy equivalence, and which is Poincaré transverse to $Q^{\prime}$.

We claim that, if $\bar{k}: X \rightarrow Y$ is the homotopy inverse of $k$, then the new splitting obstruction of

$$
\bar{k} \circ h: M \rightarrow X \rightarrow Y
$$

vanishes. Clearly the splitting obstruction of $k \circ \bar{k} \circ h$ is the same as that of $h$, but by construction this splitting obstruction differs from that of $\bar{k} \circ h$ by the $\sigma$ splitting obstruction of $k$.

Thus our new map $\bar{k} \circ h$ is $s$-cobordant to a homology manifold split map. Composing this homology manifold split map with the Poincaré transverse map $g^{\prime \prime} \circ k$ we see that our original $f$ is in fact $s$-cobordant to a homology manifold transverse map. The proof of Lemma 4.2 is thus complete.

This also concludes the proof of Theorem 4.1.

\section{DUAl transVersality FOR HOMOLOGY MANIFOLDS}

We now extend the results of Section 4 on transversality to a codimension $q$ bundle subspace $Q \subset P$ for a map $f: M^{m} \rightarrow P$ from an $m$-dimensional homology manifold with $m-q \geq 7$, and obtain dual transversality for a map $f: M^{m} \rightarrow|K|$ to the polyhedron of a $k$-dimensional simplicial complex $K$ with $m-k \geq 7$. In Section 6 we shall formulate an obstruction for a map $f: M^{m} \rightarrow|K|$ to be bordant to a dual transverse map - the obstruction is 0 for $m-k \geq 7$.

Let $X$ be a simple $m$-dimensional Poincaré duality space with a map $g: X \rightarrow|K|$ to the polyhedron of a $k$-dimensional simplicial complex $K$.

The dual transverse homology manifold structure set $\mathcal{S}^{H, K-\pitchfork}(X)$ is the set of equivalence classes of pairs $(M, h)$ with $M$ an $m$-dimensional homology manifold and $h: M \rightarrow X$ a simple homotopy equivalence such that $g h: M \rightarrow|K|$ is dual transverse, with $\left(M_{1}, h_{1}\right) \simeq\left(M_{2}, h_{2}\right)$ if there exists an $s$-cobordism $\left(W ; M_{+}, M_{2}\right)$ with a simple homotopy equivalence of the type

$$
\left(f ; h_{1}, h_{2}\right):\left(W ; M_{1}, M_{2}\right) \rightarrow X \times([0,1] ;\{0\},\{1\})
$$

such that the composite

$$
W \stackrel{f}{\rightarrow} X \times[0,1] \stackrel{\text { proj. }}{\rightarrow} X \stackrel{g}{\rightarrow}|K|
$$

is dual transverse.

Let $\mathcal{T}^{H, K-\pitchfork}(X)$ be the set of bordism classes of normal maps $(f, b): M \rightarrow X$ from $m$-dimensional homology manifolds such that $g f: X \rightarrow|K|$ is dual transverse.

Theorem 5.1. Let $m-k \geq 7$.

(i) The dual transverse homology manifold structure set fits into the surgery exact sequence

$$
\cdots \rightarrow L_{m+1}\left(\mathbb{Z}\left[\pi_{1}(X)\right]\right) \stackrel{\partial}{\rightarrow} \mathcal{S}^{K, Q-\pitchfork}(X) \stackrel{\eta}{\rightarrow} \mathcal{T}^{K, Q-\pitchfork}(X) \stackrel{\theta}{\rightarrow} L_{m}\left(\mathbb{Z}\left[\pi_{1}(X)\right]\right) .
$$

(ii) The forgetful function $\mathcal{T}^{K, Q-\pitchfork}(X) \rightarrow \mathcal{T}^{K}(X)$ is a bijection.

(iii) The forgetful function $\mathcal{S}^{K, Q-\pitchfork}(X) \rightarrow \mathcal{S}^{K}(X)$ is a bijection. In particular, $\mathcal{S}^{K, Q-\dagger}(X)$ is non-empty if and only if $\mathcal{S}^{K}(X)$ is non-empty. 
(iv) Every map $f: M \rightarrow|K|$ from an $m$-dimensional homology manifold is dual s-transverse.

Proof. Exactly as above.

\section{The DUAL TRANSVERSALITy OBSTRUCTION}

For any simplicial complex $K$ let $\Omega_{m}^{H}(K)$ (resp. $\Omega_{m}^{H \text {, } \pitchfork}(K)$ ) be the bordism group of maps $f: M \rightarrow|K|$ from $m$-dimensional homology manifolds (resp. dual transverse maps $f$ ). We now formulate the bordism obstruction to dual transversality for homology manifolds, as the failure of the forgetful map $\Omega_{m}^{H, \pitchfork}(K) \rightarrow \Omega_{m}^{H}(K)$ to be an isomorphism.

We refer to Chapters 11,12 of Ranicki [16] for an exposition of $\Delta$-sets, generalized homology theories, bordism spectra and assembly maps. The topological manifold bordism groups and the $\Omega_{\bullet}^{T O P}(\{*\})$-coefficient generalized homology groups are the homotopy groups of spectra of Kan $\Delta$-sets $\Omega_{\bullet}^{T O P}(K)$ and $\mathbb{H}_{\bullet}\left(K ; \Omega_{\bullet}^{T O P}(\{*\})\right)$ :

$$
\begin{aligned}
& \Omega_{*}^{T O P}(K)=\pi_{*}\left(\Omega_{\bullet}^{T O P}(K)\right), \\
& H_{*}\left(K ; \Omega_{\bullet}^{T O P}(\{*\})\right)=\pi_{*}\left(\mathbb{H}_{\bullet}\left(K ; \Omega_{\bullet}^{T O P}(\{*\})\right)\right) .
\end{aligned}
$$

Moreover, there is defined a topological assembly map

$$
A^{T O P}: \mathbb{H}_{\bullet}\left(K ; \Omega_{\bullet}^{T O P}(\{*\})\right) \rightarrow \Omega_{\bullet}^{T O P}(K)
$$

which is a homotopy equivalence by topological manifold transversality, inducing the Pontrjagin-Thom isomorphisms

$$
A^{T O P}: H_{*}\left(K ; \Omega_{\bullet}^{T O P}(\{*\})\right) \cong \Omega_{*}^{T O P}(K)
$$

The combinatorial construction of $\Omega_{\bullet}^{T O P}(K)$ and $A^{T O P}$ will now be extended to the homology manifold bordism spectra $\Omega_{\bullet}^{H}(K), \Omega_{\bullet}^{H, \pitchfork}(K)$ with an assembly map

$$
A^{H}: \Omega_{\bullet}^{H, \pitchfork}(K)=\mathbb{H}_{\bullet}\left(K ; \Omega_{\bullet}^{H}(\{*\})\right) \rightarrow \Omega_{\bullet}^{H}(K) .
$$

The homology assembly map $A^{H}$ is only a homotopy equivalence to the extent to which homology manifolds have transversality.

The homology manifold bordism spectrum of a simplicial complex $K$

$$
\Omega_{\bullet}^{H}(K)=\left\{\Omega_{\bullet}^{H}(K)_{m} \mid m \geq 0\right\}
$$

is the $\Omega$-spectrum with $\Omega_{\bullet}^{H}(K)_{m}$ the Kan $\Delta$-set defined by

$$
\begin{aligned}
\Omega_{\bullet}^{H}(K)_{m}^{(n)}=\{ & (m+n) \text {-dimensional homology manifold } n \text {-ads } \\
& \left(M ; \partial_{0} M, \partial_{1} M, \ldots, \partial_{n} M\right) \text { such that } \\
& \left.\partial_{0} M \cap \partial_{1} M \cap \cdots \cap \partial_{n} M=\emptyset, \text { with a map } f: M \rightarrow|K|\right\}
\end{aligned}
$$

with base simplex the empty manifold $n$-ad $\emptyset$. The homotopy groups

$$
\pi_{m}\left(\Omega_{\bullet}^{H}(K)\right)=\pi_{m-k}\left(\Omega_{\bullet}^{H}(K)_{k}\right)=\Omega_{m}^{H}(K) \quad(m \geq k \geq 0)
$$

are the bordism groups of maps $f: M \rightarrow|K|$ from closed $m$-dimensional homology manifolds. Similarly for the dual transverse bordism spectrum $\Omega_{\bullet}^{H, \pitchfork}(K)$, with the additional requirement that $f: M \rightarrow|K|$ be dual transverse. 
Proposition 6.1. The dual transverse bordism spectrum $\Omega_{*}^{H, \pitchfork}(K)$ coincides with the generalized $\Omega_{\bullet}^{H}(\{*\})$-homology spectrum of $K$,

$$
\Omega_{\bullet}^{H, \pitchfork}(K)=\mathbb{H}\left(K ; \Omega_{\bullet}^{H}(\{*\})\right),
$$

so that, on the level of homotopy groups,

$$
\Omega_{m}^{H, \pitchfork}(K)=H_{m}\left(K ; \Omega_{\bullet}^{H}(\{*\})\right) \quad(m \geq 0) .
$$

Proof. Define the generalized homology spectrum $\mathbb{H}_{\bullet}\left(K ; \Omega_{\bullet}^{H}(\{*\})\right)$ as in [16] (12.3), with an $m$-dimensional $\Omega_{\bullet}^{H}(\{*\})$-coefficient cycle $([16], 12.17)$

$$
x=\left\{M(\sigma)^{m-|\sigma|} \mid \sigma \in K\right\}
$$

essentially the same as a dual transverse map

$$
f(x): M^{m}=\bigcup_{\sigma \in K} M(\sigma) \rightarrow|K|
$$

from a closed $m$-dimensional homology manifold, with inverse images the $(m-|\sigma|)$ dimensional homology manifolds with

$$
f(x)^{-1}(D(\sigma, K), \partial D(\sigma, K))=(M(\sigma), \partial M(\sigma)) \quad(\sigma \in K) .
$$

The homotopy group

$$
\pi_{m}\left(\mathbb{H}_{\bullet}\left(K ; \Omega_{\bullet}^{H}(\{*\})\right)\right)=H_{m}\left(K ; \Omega_{\bullet}^{H}(\{*\})\right)
$$

is the cobordism group of such cycles, and is the bordism group of dual transverse maps $f: M \rightarrow|K|$ from $m$-dimensional homology manifolds.

The homology assembly map of spectra

$$
A^{H}: \Omega_{\bullet}^{H, \pitchfork}(K)=\mathbb{H}_{\bullet}\left(K ; \Omega_{\bullet}^{H}(\{*\})\right) \rightarrow \Omega_{\bullet}^{H}(K)
$$

is defined as in [16] (12.18), inducing on the level of homotopy groups the assembly maps of bordism groups

$$
A^{H}: \Omega_{*}^{H, \pitchfork}(K)=H_{*}\left(K ; \Omega_{\bullet}^{H}(\{*\})\right) \rightarrow \Omega_{*}^{H}(K)
$$

which forget dual transversality.

Definition 6.2. Given an $m$-dimensional homology manifold

$$
M=\bigcup_{\alpha} M_{\alpha}
$$

with $M_{\alpha}$ the components of $M$, set

$$
\mathcal{I}(M)=\sum_{\alpha}\left[M_{\alpha}\right] i\left(M_{\alpha}\right) \in H_{m}(M)\left[L_{0}(\mathbb{Z})\right],
$$

with $\left[M_{\alpha}\right] \in H_{m}(M)$ the image of the fundamental class $\left[M_{\alpha}\right] \in H_{m}\left(M_{\alpha}\right)$.

The augmentation map

$$
H_{m}(M)\left[L_{0}(\mathbb{Z})\right] \rightarrow H_{m}\left(M ; L_{0}(\mathbb{Z})\right)=H_{m}(M) \otimes_{\mathbb{Z}} L_{0}(\mathbb{Z}) ; x[y] \rightarrow x \otimes y
$$

sends $\mathcal{I}(M)$ to the resolution obstruction of $M$,

$$
i(M)=\sum_{\alpha} i\left(M_{\alpha}\right) \in H_{m}\left(M ; L_{0}(\mathbb{Z})\right)=\sum_{\alpha} L_{0}(\mathbb{Z}) .
$$

We shall now verify that $\mathcal{I}(M, f)$ and $\widetilde{\mathcal{I}}(M, f)$ are bordism invariants: 
Proposition 6.3. Let $f: M \rightarrow|K|$ be a map from an m-dimensional homology manifold $M$ to a polyhedron, and let $M=\bigcup_{\alpha} M_{\alpha}$ be the decomposition of $M$ into components with $f_{\alpha}=f\left|: M_{\alpha} \rightarrow\right| K \mid$.

(i) The element

$$
\mathcal{I}(M, f)=\sum_{\alpha}\left(f_{\alpha}\right)_{*}\left[M_{\alpha}\right]\left[i\left(M_{\alpha}\right)\right] \in H_{m}(K)\left[L_{0}(\mathbb{Z})\right]
$$

is a homology manifold bordism invariant.

(ii) If $f$ is a dual transverse map, then

$$
\mathcal{I}(M, f)=0 \in H_{m}(K)\left[L_{0}(\mathbb{Z})\right] .
$$

(iii) If $f_{*}: H_{m}(M) \rightarrow H_{m}(K)$ is an isomorphism and $f$ is homology manifold bordant to a dual transverse map, then

$$
i(M)=0 \in H_{m}\left(M ; L_{0}(\mathbb{Z})\right) .
$$

Proof. (i) Given a bordism

$$
\left(g ; f, f^{\prime}\right):\left(W ; M, M^{\prime}\right) \rightarrow|K|,
$$

denote the connected components by

$$
\left(g_{\alpha} ; f_{\alpha}, f_{\alpha}^{\prime}\right):\left(W_{\alpha} ; M_{\alpha}, M_{\alpha}^{\prime}\right) \rightarrow|K| .
$$

We have

$$
\left(f_{\alpha}\right)_{*}\left[M_{\alpha}\right]=\left(f_{\alpha}^{\prime}\right)_{*}\left[M_{\alpha}^{\prime}\right] \in H_{m}(K),
$$

as usual. Furthermore,

$$
i\left(M_{\alpha}\right)=i\left(W_{\alpha}\right)=i\left(M_{\alpha}^{\prime}\right),
$$

because $i(X)=i(\partial X)$ for any connected homology manifold with non-empty boundary (Quinn [12], 1.1). Thus we have

$$
\begin{aligned}
\mathcal{I}(M, f) & =\sum_{\alpha}\left(f_{\alpha}\right)_{*}\left[M_{\alpha}\right]\left[i\left(M_{\alpha}\right)\right] \\
& =\sum_{\alpha^{\prime}}\left(f_{\alpha^{\prime}}^{\prime}\right)_{*}\left[i\left(M_{\alpha^{\prime}}\right)\right]=\mathcal{I}\left(M^{\prime}, f^{\prime}\right) \in H_{m}(K)\left[L_{0}(\mathbb{Z})\right] .
\end{aligned}
$$

(ii) The augmentation map

$$
H_{m}(K)\left[L_{0}(\mathbb{Z})\right] \rightarrow H_{m}\left(K ; L_{0}(\mathbb{Z})\right)=H_{m}(K) \otimes_{\mathbb{Z}} L_{0}(\mathbb{Z}) ; x[y] \rightarrow x \otimes y
$$

sends $\mathcal{I}(M, f)$ to $f_{*} i(M) \in H_{m}\left(K ; L_{0}(\mathbb{Z})\right)$, for any map $f: M \rightarrow|K|$. If $M$ is connected, then $i(M) \in L_{0}(\mathbb{Z})$ and

$$
\mathcal{I}(M, f)=f_{*}[M][i(M)] \in H_{m}(K)[i(M)] \subset H_{m}(K)\left[L_{0}(\mathbb{Z})\right],
$$

so that $f_{*} i(M)=0$ implies $\mathcal{I}(M, f)=0$. If $f: M \rightarrow|K|$ is dual transverse, then

$$
f_{*} i(M)=\sum_{\sigma \in K^{(m)}} i\left(f^{-1} D(\sigma, K)\right) \sigma \in H_{m}\left(K ; L_{0}(\mathbb{Z})\right) .
$$

Each $f^{-1} D(\sigma, K)$ is a 0-dimensional homology manifold, which is a disjoint union of points, with resolution obstruction $i\left(f^{-1} D(\sigma, K)\right)=0$, so that $f_{*} i(M)=0$. Thus, if $M$ is connected and $f: M \rightarrow|K|$ is dual transverse, then $f_{*} i(M)=0$, and hence $\mathcal{I}(M, f)=0$. Apply this to each component of $M$.

(iii) Combine (i) and (ii). 
Definition 6.4. (i) For any space $X$ define a morphism

$$
\mathcal{I}: \Omega_{m}^{H}(X) \rightarrow H_{m}(X)\left[L_{0}(\mathbb{Z})\right] ;(M, f) \mapsto \mathcal{I}(M, f)=f_{*} \mathcal{I}(M)
$$

such that

$$
i: \Omega_{m}^{H}(X) \stackrel{\mathcal{I}}{\rightarrow} H_{m}(X)\left[L_{0}(\mathbb{Z})\right] \rightarrow H_{m}\left(X ; L_{0}(\mathbb{Z})\right) ;(M, f) \mapsto f_{*} i(M)
$$

and

$$
\Omega_{m}^{H}(X) \stackrel{\mathcal{I}}{\longrightarrow} H_{m}(X)\left[L_{0}(\mathbb{Z})\right] \stackrel{\text { proj. }}{\longrightarrow} H_{m}(X) ;(M, f) \mapsto f_{*}[M]
$$

(ii) Let

$$
\widetilde{L}_{0}(\mathbb{Z})=L_{0}(\mathbb{Z}) \backslash\{0\},
$$

and let

$$
\tilde{\mathcal{I}}: \Omega_{m}^{H}(X) \stackrel{\mathcal{I}}{\rightarrow} H_{m}(X)\left[L_{0}(\mathbb{Z})\right] \rightarrow H_{m}(X)\left[\widetilde{L}_{0}(\mathbb{Z})\right] .
$$

For any $\Omega$-spectrum $X_{\bullet}=\left\{X_{n} \mid n \geq 0\right\}$ of $\Delta$-sets there is defined an $\Omega$-spectrum of $\Delta$-sets

$$
X_{\bullet}\left[L_{0}(\mathbb{Z})\right]=\left\{X_{n}\left[L_{0}(\mathbb{Z})\right] \mid n \geq 0\right\}
$$

with

$$
\pi_{m}\left(X\left[L_{0}(\mathbb{Z})\right]\right)=\pi_{m}(X)\left[L_{0}(\mathbb{Z})\right](m \geq 0) .
$$

Similarly for $\widetilde{L}_{0}(\mathbb{Z})$.

Proposition 6.5. Let $K$ be a $k$-dimensional simplicial complex.

(i) The map of $\Omega$-spectra

$$
\Omega_{\bullet}^{H}(K) \rightarrow \Omega_{\bullet}^{T O P}(K)\left[L_{0}(\mathbb{Z})\right] ; M \mapsto M_{T O P}[i(M)]
$$

induces maps

$$
\pi_{m}\left(\Omega_{\bullet}^{H}(K)\right)=\Omega_{m}^{H}(K) \rightarrow \pi_{m}\left(\Omega_{\bullet}^{T O P}(K)\left[L_{0}(\mathbb{Z})\right]\right)=\Omega_{m}^{T O P}(K)\left[L_{0}(\mathbb{Z})\right]
$$

which are isomorphisms for $m \geq 6$.

(ii) The composite

$$
\Omega_{m}^{H, \pitchfork}(K) \stackrel{A^{H}}{\rightarrow} \Omega_{m}^{H}(K) \stackrel{\tilde{\mathcal{I}}}{\rightarrow} H_{m}(K)\left[\widetilde{L}_{0}(\mathbb{Z})\right]
$$

is 0 .

(iii) The homology assembly map

$$
A^{H}: \Omega_{m}^{H, \pitchfork}(K)=H_{m}\left(K ; \Omega_{\bullet}^{H}(\{*\})\right) \rightarrow \Omega_{m}^{H}(K)
$$

is an isomorphism for $m-k \geq 6$.

Proof. (i) See Johnston [6] (cf. Corollary [1.6).

(ii) By Proposition 6.3

$$
\operatorname{im}\left(\mathcal{I} A^{H}: \Omega_{m}^{H, \pitchfork}(K) \rightarrow H_{m}(K)\left[L_{0}(\mathbb{Z})\right]\right) \subseteq H_{m}(K)[0] \subseteq H_{m}(K)\left[L_{0}(\mathbb{Z})\right],
$$

so that $\widetilde{\mathcal{I}} A^{H}=0$.

(iii) By (i) we know that the maps of Kan $\Delta$-sets

$$
\Omega_{\bullet}^{H}(\{*\})_{n} \rightarrow \Omega_{\bullet}^{T O P}(\{*\})_{n}\left[L_{0}(\mathbb{Z})\right] ; M \mapsto M_{T O P}[i(M)],
$$

are homotopy equivalences for $n \geq 6$. It follows that for $m \geq k+6$

$$
\mathbb{H}\left(K ; \Omega_{\bullet}^{H}(\{*\})\right)_{m} \simeq \mathbb{H}\left(K ; \Omega_{\bullet}^{T O P}(\{*\})\right)_{m}\left[L_{0}(\mathbb{Z})\right]
$$


and hence that

$$
\begin{aligned}
H_{m}\left(K ; \Omega_{\bullet}^{H}(\{*\})\right) & =\pi_{0}\left(\mathbb{H}\left(K ; \Omega_{\bullet}^{H}(\{*\})\right)_{m}\right) \\
& =\pi_{0}\left(\mathbb{H}\left(K ; \Omega_{\bullet}^{T O P}(\{*\})\right)_{m}\left[L_{0}(\mathbb{Z})\right]\right) \\
& =H_{m}\left(K ; \Omega_{\bullet}^{T O P}(\{*\})\right)\left[L_{0}(\mathbb{Z})\right] \\
& =\Omega_{m}^{T O P}(K)\left[L_{0}(\mathbb{Z})\right]=\Omega_{m}^{H}(K) .
\end{aligned}
$$

Theorem 6.6. (i) The composite

$$
\Omega_{m}^{H, \pitchfork}(K) \rightarrow \Omega_{m}^{H}(K) \stackrel{\widetilde{\mathcal{I}}}{\rightarrow} H_{m}(K)\left[\widetilde{L}_{0}(\mathbb{Z})\right]
$$

is 0 . Thus if $f: M \rightarrow|K|$ is homology manifold bordant to a dual transverse map then

$$
\mathcal{I}(M, f) \in H_{m}(K)[0] \subset H_{m}(K)\left[L_{0}(\mathbb{Z})\right],
$$

and either $f_{*}[M]=0 \in H_{m}(K)$ or $i(M)=0 \in H_{m}\left(M ; L_{0}(\mathbb{Z})\right)$, but in any case

$$
f_{*} i(M)=0 \in H_{m}\left(K ; L_{0}(\mathbb{Z})\right) .
$$

(ii) There exists a spectrum $\mathcal{L} \bullet$ whose homotopy groups fit into an exact sequence $\cdots \rightarrow \Omega_{m}^{H}(\{*\}) \rightarrow \Omega_{m}^{T O P}(\{*\})\left[L_{0}(\mathbb{Z})\right] \rightarrow \pi_{m}\left(\mathcal{L}_{\bullet}\right) \rightarrow \Omega_{m-1}^{H}(\{*\}) \rightarrow \ldots$,

with

$$
\pi_{m}(\mathcal{L} \bullet)= \begin{cases}\mathbb{Z}\left[\widetilde{L}_{0}(\mathbb{Z})\right] & \text { if } m=0 \\ 0 & \text { if } m \geq 1 \text { and } m \neq 4,5\end{cases}
$$

and

$$
\begin{aligned}
\Omega_{m}^{H}(K) \rightarrow \Omega_{m}^{T O P}(K)\left[L_{0}(\mathbb{Z})\right] & \stackrel{\text { I }}{\rightarrow} H_{m}(K ; \mathcal{L} \bullet) \\
& \rightarrow H_{m}\left(K ; \pi_{0}(\mathcal{L} \bullet)\right)=H_{m}(K)\left[\widetilde{L}_{0}(\mathbb{Z})\right] ; \\
(M, f: M \rightarrow|K|) & \mapsto f_{*}[M][\overline{i(M)]} .
\end{aligned}
$$

(iii) For $m \geq 6$ there is defined an exact sequence

$$
\cdots \rightarrow \Omega_{m}^{H, \pitchfork}(K) \stackrel{A^{H}}{\rightarrow} \Omega_{m}^{H}(K) \stackrel{\widetilde{\mathcal{I}}}{\rightarrow} H_{m}(K ; \mathcal{L} \bullet) \rightarrow \Omega_{m-1}^{H, \pitchfork}(K) \rightarrow \ldots
$$

The proof of 6.6 (i) is given by Proposition 6.3 We now give the proofs of 6.6 (ii) and (iii). The spectrum $\mathcal{L}_{\bullet}$ in (ii) is given by

Definition 6.7. Let

$$
\mathcal{L}_{\bullet}=\operatorname{cofibre}\left(\Omega_{\bullet}^{H}(\{*\}) \rightarrow \Omega_{\bullet}^{T O P}(\{*\})\left[L_{0}(\mathbb{Z})\right]\right),
$$

an $\Omega$-spectrum whose homotopy groups fit into an exact sequence

$$
\cdots \rightarrow \Omega_{m}^{H}(\{*\}) \rightarrow \Omega_{m}^{T O P}(\{*\})\left[L_{0}(\mathbb{Z})\right] \rightarrow \pi_{m}(\mathcal{L} \bullet) \rightarrow \Omega_{m-1}^{H}(\{*\}) \rightarrow \ldots
$$

It is now immediate from the identities

$$
\begin{aligned}
& \Omega_{m}^{H}(\{*\})= \begin{cases}\mathbb{Z} & \text { if } m=0, \\
0 & \text { if } m=1,2, \\
\Omega_{m}^{T O P}(\{*\})\left[L_{0}(\mathbb{Z})\right] & \text { if } m \geq 6,\end{cases} \\
& \Omega_{m}^{T O P}(\{*\})= \begin{cases}\mathbb{Z} & \text { if } m=0, \\
0 & \text { if } m=1,2,3,\end{cases}
\end{aligned}
$$


that

$$
\pi_{m}(\mathcal{L} \bullet)= \begin{cases}\mathbb{Z}\left[\widetilde{L}_{0}(\mathbb{Z})\right] & \text { if } m=0, \\ 0 & \text { if } m \geq 1 \text { and } m \neq 4,5,\end{cases}
$$

as claimed in Theorem 6.6 (ii).

The exact sequence in the statement of Theorem 6.6 (iii) is given by

Proposition 6.8. For $m \geq 6$ there is defined an exact sequence

$$
\cdots \rightarrow \Omega_{m}^{H, \pitchfork}(K) \stackrel{A^{H}}{\rightarrow} \Omega_{m}^{H}(K) \stackrel{\widetilde{\mathcal{I}}}{\rightarrow} H_{m}(K ; \mathcal{L} \bullet) \rightarrow \Omega_{m-1}^{H, \pitchfork}(K) \rightarrow \ldots .
$$

Proof. This is just the exact sequence

$$
\begin{aligned}
\cdots \rightarrow H_{m}\left(K ; \Omega_{\bullet}^{H}(\{*\})\right) & \rightarrow H_{m}\left(K ; \Omega_{\bullet}^{T O P}(\{*\})\left[L_{0}(\mathbb{Z})\right]\right) \\
& \rightarrow H_{m}(K ; \mathcal{L} \bullet) \rightarrow H_{m-1}\left(K ; \Omega_{\bullet}^{H}(\{*\})\right) \rightarrow \ldots
\end{aligned}
$$

induced by the cofibration sequence of spectra

$$
\Omega_{\bullet}^{H}(\{*\}) \rightarrow \Omega_{\bullet}^{T O P}(\{*\})\left[L_{0}(\mathbb{Z})\right] \rightarrow \mathcal{L}_{\bullet},
$$

using 1.6 and 6.1 to identify

$$
\begin{aligned}
& \Omega_{m}^{H, \pitchfork}(K)=H_{m}\left(K ; \Omega_{\bullet}^{H}(\{*\})\right), \\
& \Omega_{m}^{H}(K)=H_{m}\left(K ; \Omega_{\bullet}^{T O P}(\{*\})\right)\left[L_{0}(\mathbb{Z})\right] .
\end{aligned}
$$

This also completes the proof of Theorem 6.6

In the special case $K=S^{m}$ we have

Corollary 6.9. (i) A map $f: M^{m} \rightarrow S^{m}$ from an m-dimensional homology manifold $M$ determines the element

$$
\widetilde{\mathcal{I}}(M, f)=\operatorname{degree}(f)[i(M)] \in \mathbb{Z}\left[\widetilde{L}_{0}(\mathbb{Z})\right]
$$

which vanishes if (and for $m \geq 6$ only if) $f$ is bordant to a dual transverse map.

(ii) For connected $M, \widetilde{\mathcal{I}}(M, f)=0$ if and only if either degree $(f)=0 \in \mathbb{Z}$ or $i(M)=0 \in H_{m}\left(M ; L_{0}(\mathbb{Z})\right)=L_{0}(\mathbb{Z})$.

Proof. This follows from the sequence of 6.6 (i),

$$
H_{m}\left(S^{m} ; \Omega_{\bullet}^{H}(\{*\})\right) \stackrel{A^{H}}{\longrightarrow} \Omega_{m}^{H}\left(S^{m}\right) \stackrel{\widetilde{\mathcal{I}}}{\longrightarrow} \mathbb{Z}\left[\widetilde{L}_{0}(\mathbb{Z})\right],
$$

whose composite is 0 , and which is exact for $m \geq 6$. By a simple calculation,

$$
H_{m}\left(S^{m} ; \Omega_{\bullet}^{H}(\{*\})\right)=\Omega_{0}^{H}(\{*\}) \oplus \Omega_{m}^{H}(\{*\}) .
$$

Since any zero-dimensional homology manifold is a disjoint union of points,

$$
\Omega_{0}^{H}(\{*\})=\Omega_{0}^{T O P}(\{*\})=\mathbb{Z} .
$$

By 1.6

$$
\Omega_{m}^{H}(\{*\})=\Omega_{m}^{T O P}(\{*\})\left[L_{0}(\mathbb{Z})\right] \quad(m \geq 6),
$$

so that

$$
H_{m}\left(S^{m} ; \Omega_{\bullet}^{H}(\{*\})\right)=\Omega_{0}^{T O P}(\{*\}) \oplus \Omega_{m}^{T O P}(\{*\})\left[L_{0}(\mathbb{Z})\right] .
$$

Also

$$
\Omega_{m}^{H}\left(S^{m}\right)=\Omega_{m}^{T O P}\left(S^{m}\right)\left[L_{0}(\mathbb{Z})\right] \quad(m \geq 6),
$$


and by topological transversality

$$
\Omega_{m}^{T O P}\left(S^{m}\right)=H_{m}\left(S^{m} ; \Omega_{\bullet}^{T O P}(\{*\})\right)=\Omega_{0}^{T O P}(\{*\}) \oplus \Omega_{m}^{T O P}(\{*\}),
$$

so that

$$
\Omega_{m}^{H}\left(S^{m}\right)=\Omega_{0}^{T O P}(\{*\})\left[L_{0}(\mathbb{Z})\right] \oplus \Omega_{m}^{T O P}(\{*\})\left[L_{0}(\mathbb{Z})\right] \quad(m \geq 6) .
$$

The components of the assembly map

$$
\begin{aligned}
& A^{H}=A_{0}^{H} \oplus A_{m}^{H}: \\
& \Omega_{0}^{H}(\{*\}) \oplus \Omega_{m}^{H}(\{*\}) \rightarrow \Omega_{0}^{T O P}(\{*\})\left[L_{0}(\mathbb{Z})\right] \oplus \Omega_{m}^{T O P}(\{*\})\left[L_{0}(\mathbb{Z})\right]
\end{aligned}
$$

are given by the inclusion

$$
A_{0}^{H}: \Omega_{0}^{H}(\{*\})=\mathbb{Z} \rightarrow \Omega_{0}^{T O P}(\{*\})\left[L_{0}(\mathbb{Z})\right]=\mathbb{Z}\left[L_{0}(\mathbb{Z})\right] ; t \mapsto t[0]
$$

and the isomorphism

$$
A_{m}^{H}: \Omega_{m}^{H}(\{*\}) \cong \Omega_{m}^{T O P}(\{*\})\left[L_{0}(\mathbb{Z})\right] .
$$

The cokernel of $A^{H}$ is thus given by the cokernel of $A_{0}^{H}$ as $\mathbb{Z}\left[\widetilde{L}_{0}(\mathbb{Z})\right]$. In particular, if $m \geq 6$ and $\Sigma^{m}$ is one of the $m$-dimensional homology spheres with

$$
i\left(\Sigma^{m}\right) \neq 0 \in H_{m}\left(\Sigma^{m} ; L_{0}(\mathbb{Z})\right)=L_{0}(\mathbb{Z})
$$

constructed in [1, then there exists a homotopy equivalence $f: \Sigma^{m} \rightarrow S^{m}$ with

$$
\widetilde{\mathcal{I}}\left(\Sigma^{m}, f\right)=i\left(\Sigma^{m}\right) \neq 0 \in \mathbb{Z}\left[\widetilde{L}_{0}(\mathbb{Z})\right],
$$

so that $f$ is not bordant to a dual transverse map.

Every compact $A N R X$ is simple homotopy equivalent to a polyhedron $|K|$, by the result of West [21]. However, if $X$ is an $m$-dimensional homology manifold then $|K|$ need not be a homology manifold.

Theorem (Levitt and Ranicki 9], Ranicki [16] (19.6)). Let X be a compact ANR which is a simple $m$-dimensional Poincaré space, and let $f: X \rightarrow|K|$ be a simple homotopy equivalence to a polyhedron. The map $f$ is simple Poincaré bordant to a dual Poincaré transverse map $f^{\prime}: X^{\prime} \rightarrow|K|$ if (and for $m \geq 6$ only if) $X$ is simple homotopy equivalent to a topological manifold.

Theorem 6.6 will now be used to obtain an analogous characterization of resolvable homology manifolds.

Proposition 6.10. Let $M$ be an m-dimensional homology manifold, and let $f$ : $M \rightarrow|K|$ be a simple homotopy equivalence to a polyhedron. The map $f$ is $s$ cobordant to a dual transverse homotopy equivalence $f^{\prime}: M^{\prime} \rightarrow|K|$ if (and for $m \geq 6$ only if ) $M$ is resolvable.

Proof. If $M$ is resolvable, then the mapping cylinder of a resolution $M_{T O P} \rightarrow M$ is an $s$-cobordism $\left(W ; M, M_{T O P}\right)$ with a bordism

$$
\left(g ; f, f_{T O P}\right):\left(W ; M, M_{T O P}\right) \rightarrow|K|
$$

to a homotopy equivalence $f_{T O P}: M_{T O P} \rightarrow|K|$ which is topologically dual transverse.

Conversely, suppose that $f: M \rightarrow|K|$ is a dual transverse homotopy equivalence. Without loss of generality, it may be assumed that $M$ is connected, so that

$$
\mathcal{I}(M)=[i(M)] \in \mathbb{Z}[i(M)] \subseteq H_{m}(M)\left[L_{0}(\mathbb{Z})\right]=\mathbb{Z}\left[L_{0}(\mathbb{Z})\right]
$$


The composite

$$
\Omega_{m}^{H}(K) \stackrel{\widetilde{\mathcal{I}}}{\rightarrow} H_{m}(K ; \mathcal{L} \bullet) \rightarrow H_{m}(K)\left[\widetilde{L}_{0}(\mathbb{Z})\right]
$$

sends $(M, f)$ to

$$
\widetilde{\mathcal{I}}(M, f)= \begin{cases}{[i(M)] \in \mathbb{Z}[i(M)] \subseteq \mathbb{Z}\left[\widetilde{L}_{0}(\mathbb{Z})\right]} & \text { if } i(M) \neq 0, \\ 0 \in \mathbb{Z}\left[\widetilde{L}_{0}(\mathbb{Z})\right] & \text { if } i(M)=0 .\end{cases}
$$

Since $f: M \rightarrow|K|$ is dual transverse, $i(M)=0 \in L_{0}(\mathbb{Z})$ by 6.6 (ii).

\section{The multiplicative StRuCture of Homology MANifold BORDism}

The product of an $m$-dimensional Poincaré space $X$ and an $n$-dimensional Poincaré space $Y$ is an $(m+n)$-dimensional Poincaré space $X \times Y$, with Spivak normal fibration

$$
\nu_{X \times Y}=\nu_{X} \times \nu_{Y}: X \times Y \rightarrow B G .
$$

If $X$ is an $m$-dimensional homology manifold and $Y$ is an $n$-dimensional homology manifold, then $X \times Y$ is an $(m+n)$-dimensional homology manifold, with the resolution obstructions satisfying the index product formula of Quinn 12 .

$$
1+8 i(X \times Y)=(1+8 i(X))(1+8 i(Y)) \in 1+8 \mathbb{Z} .
$$

In general, the canonical TOP reductions of the Spivak normal fibrations of $X, Y$, $X \times Y$ are such that

$$
\widetilde{\nu}_{X \times Y} \neq \widetilde{\nu}_{X} \times \widetilde{\nu}_{Y}: X \times Y \rightarrow B T O P .
$$

We shall now analyze the difference

$$
\widetilde{\nu}_{X \times Y}-\widetilde{\nu}_{X} \times \widetilde{\nu}_{Y}: X \times Y \rightarrow G / T O P
$$

using the multiplicative properties of the algebraic $L$-spectra of [16]. This analysis will be used to obtain the product structure on $\Omega_{*}^{T O P}(K)\left[L_{0}(\mathbb{Z})\right]$ which corresponds to the product structure on $\Omega_{*}^{H}$,

$$
\Omega_{m}^{H}(J) \otimes \Omega_{n}^{H}(K) \rightarrow \Omega_{m+n}^{H}(J \times K) ; X \otimes Y \mapsto X \times Y,
$$

under the isomorphism of abelian groups given by 1.6 ,

$$
\Omega_{*}^{H}(K) \stackrel{\cong}{\rightrightarrows} \Omega_{*}^{T O P}(K)\left[L_{0}(\mathbb{Z})\right] ; X \mapsto X_{T O P}[i(X)] .
$$

The Spivak normal fibration $\nu_{X}: X \rightarrow B G(k)$ ( $k$ large) of an $m$-dimensional Poincaré space $X$ is equipped with a degree 1 map $\rho: S^{m+k} \rightarrow T\left(\nu_{X}\right)$. If $\nu_{X}$ is $T O P$ reducible then for any TOP reduction $\widetilde{\nu}_{X}: X \rightarrow B T O P$ the Browder-Novikov transversality construction gives a degree 1 normal map

$$
f=\rho \mid: M=\rho^{-1}(X) \rightarrow X
$$

with $M$ an $m$-dimensional topological manifold. If $\widetilde{\nu}, \widetilde{\nu}^{\prime}: X \rightarrow B T O P$ are two TOP reductions of $\nu_{X}$, then the difference is classified by an element

$$
t\left(\widetilde{\nu}, \widetilde{\nu}^{\prime}\right) \in H_{m}\left(X ; \mathbb{L}_{\bullet}\right)=[X, G / T O P]
$$

and the corresponding degree 1 normal maps $f: M \rightarrow X, f^{\prime}: M^{\prime} \rightarrow X$ can be chosen so that $f^{\prime}=f g$ for a degree 1 normal map $g: M^{\prime} \rightarrow M$ classified by an element

$$
[g]_{\mathbb{L}} \in H_{m}\left(M ; \mathbb{L}_{\bullet}\right)=[M, G / T O P]
$$


such that $f_{*}[g]_{\mathbb{L}}=t\left(\widetilde{\nu}, \widetilde{\nu}^{\prime}\right)$. The surgery obstruction of $g$ is the assembly of $[g]_{\mathbb{L}}$,

$$
\sigma_{*}(g)=A\left([g]_{\mathbb{L}}\right) \in L_{m}\left(\mathbb{Z}\left[\pi_{1}(M)\right]\right),
$$

and the surgery obstructions of $f$ and $f^{\prime}$ differ by

$$
\sigma_{*}\left(f^{\prime}\right)-\sigma_{*}(f)=A\left(f_{*}[g]_{\mathbb{L}}\right)=A\left(t\left(\widetilde{\nu}, \widetilde{\nu}^{\prime}\right)\right) \in L_{m}\left(\mathbb{Z}\left[\pi_{1}(X)\right]\right) .
$$

See Chapter 16 of [16] for the $L$-theory orientation of topology.

Let $\mathbb{L}^{\bullet}$ be the 0 -connective symmetric ring $L$-spectrum of $\mathbb{Z}$, with homotopy groups

$$
\pi_{m}\left(\mathbb{L}^{\bullet}\right)=L^{m}(\mathbb{Z})= \begin{cases}\mathbb{Z} & \text { if } m \equiv 0(\bmod 4), \\ \mathbb{Z}_{2} & \text { if } m \equiv 1(\bmod 4), \\ 0 & \text { if } m \equiv 2(\bmod 4), \\ 0 & \text { if } m \equiv 3(\bmod 4)\end{cases}
$$

Theorem 7.1 (Ranicki [16], 25.7). An m-dimensional homology manifold X has a canonical $\mathbb{L} \bullet$-orientation

$$
[X]_{\mathbb{L}} \in H_{m}\left(X ; \mathbb{L}^{\bullet}\right)
$$

with assembly the symmetric signature of $X$,

$$
A\left([X]_{\mathbb{L}}\right)=\sigma^{*}(X) \in L^{m}\left(\mathbb{Z}\left[\pi_{1}(X)\right]\right),
$$

and such that there are $\mathbb{L}^{\bullet}$-coefficient Poincaré duality isomorphisms

$$
[X]_{\mathbb{L}} \cap-: H^{*}\left(X ; \mathbb{L}^{\bullet}\right) \cong H_{m-*}\left(X ; \mathbb{L}^{\bullet}\right)
$$

as well as with coefficients in any $\mathbb{L}^{\bullet}$-module spectrum $\left(\right.$ e.g. $\left.\mathbb{L}_{\bullet}, \overline{\mathbb{L}}_{\bullet}\right)$.

Proof. See [16] (16.16) for the canonical $\mathbb{L} \bullet$-orientation of an $m$-dimensional topological manifold. Let $f: M=X_{T O P} \rightarrow X$ be the normal map from a topological manifold determined (up to normal bordism) by the canonical TOP reduction $\widetilde{\nu}_{X}$ of $\nu_{X}$, with surgery obstruction

$$
\sigma_{*}(f)=\bar{A}\left([f]_{\mathbb{L}}\right)=\bar{A}(-i(X)) \in L_{m}\left(\mathbb{Z}\left[\pi_{1}(X)\right]\right)
$$

the assembly of

$$
[f]_{\mathbb{L}}=(-i(X), 0) \in H_{m}\left(X ; \overline{\mathbb{L}}_{\bullet}\right)=H_{m}\left(X ; L_{0}(\mathbb{Z})\right) \oplus H_{m}\left(X ; \mathbb{L}_{\bullet}\right)
$$

The canonical $\mathbb{L} \bullet$-theory orientation of $X$ is given by

$$
\begin{aligned}
{[X]_{\mathbb{L}} } & =f_{*}[M]_{\mathbb{L}}-(1+T)[f]_{\mathbb{L}}=f_{*}[M]_{\mathbb{L}}+(8 i(X), 0) \\
& \in H_{m}\left(X ; \mathbb{L}^{\bullet}\right)=H_{m}\left(X ; L^{0}(\mathbb{Z})\right) \oplus H_{m}(X ; \mathbb{L} \bullet\langle 1\rangle) .
\end{aligned}
$$

Let $\mathbb{L} \bullet\langle 1\rangle$ be the 1 -connective cover of $\mathbb{L} \bullet$, so that for any space $X$ there is defined an exact sequence

$$
\cdots \rightarrow H_{m}\left(X ; \mathbb{L}^{\bullet}\langle 1\rangle\right) \rightarrow H_{m}\left(X ; \mathbb{L}^{\bullet}\right) \rightarrow H_{m}\left(X ; L^{0}(\mathbb{Z})\right) \rightarrow H_{m-1}\left(X ; \mathbb{L}_{\bullet}\langle 1\rangle\right) \rightarrow \ldots
$$

The 0 th spaces of $\mathbb{L}^{\bullet}$ and $\mathbb{L} \bullet\langle 1\rangle$ are related by

$$
\mathbb{L}^{0}=L^{0}(\mathbb{Z}) \times \mathbb{L}^{0}\langle 1\rangle,
$$


so that for an $m$-dimensional homology manifold $X$

$$
\begin{aligned}
H_{m}\left(X ; \mathbb{L}^{\bullet}\right) & \cong H^{0}\left(X ; \mathbb{L}^{\bullet}\right) \\
& \cong\left[X, \mathbb{L}^{0}\right] \cong\left[X, L^{0}(\mathbb{Z}) \times \mathbb{L}^{0}\langle 1\rangle\right] \\
& \cong H^{0}\left(X ; L^{0}(\mathbb{Z})\right) \oplus H^{0}(X ; \mathbb{L} \bullet\langle 1\rangle) \\
& \cong H_{m}\left(X ; L^{0}(\mathbb{Z})\right) \oplus H_{m}(X ; \mathbb{L} \bullet\langle 1\rangle) .
\end{aligned}
$$

For a connected $X$ we have

$$
[X]_{\mathbb{L}}=\left(1+8 i(X), \widetilde{[X]_{\mathbb{L}}}\right) \in H_{m}\left(X ; \mathbb{L}^{\bullet}\right)=H_{m}\left(X ; L^{0}(\mathbb{Z})\right) \oplus H_{m}(X ; \mathbb{L} \bullet\langle 1\rangle),
$$

writing the ordinary fundamental class as $[X]=1 \in H_{m}\left(X ; L^{0}(\mathbb{Z})\right)=\mathbb{Z}$.

Corollary 7.2. Given an $m$-dimensional homology manifold $X$, let $f: M=X_{T O P}$ $\rightarrow X$ be a normal map from a topological manifold in the canonical class. The canonical $\mathbb{L} \bullet$-orientation of $M$ is such that

$$
\left.[M]_{\mathbb{L}}=(1, \widetilde{[M}]_{\mathbb{L}}\right) \in H_{m}\left(M ; \mathbb{L}^{\bullet}\right)=H_{m}\left(M ; L^{0}(\mathbb{Z})\right) \oplus H_{m}(M ; \mathbb{L} \bullet\langle 1\rangle)
$$

with

$$
\begin{aligned}
f_{*}[M]_{\mathbb{L}} & =-8 i(X)+[X]_{\mathbb{L}} \in H_{m}\left(X ; \mathbb{L}^{\bullet}\right), \\
f_{*} \widetilde{[M]_{\mathbb{L}}} & =\widetilde{[X]_{\mathbb{L}}} \in H_{m}(X ; \mathbb{L} \bullet\langle 1\rangle) .
\end{aligned}
$$

Definition 7.3. Let $X$ be an $m$-dimensional homology manifold, and let $\widetilde{\nu}_{X}: X \rightarrow$ $B T O P$ be the canonical Ferry-Pedersen 4] TOP reduction of the Spivak normal fibration $\nu_{X}: X \rightarrow B G$, with stable inverse $-\widetilde{\nu}_{X}: X \rightarrow B T O P$.

(i) The (rational) Pontrjagin classes of $X$ are the Pontrjagin classes of $-\widetilde{\nu}_{X}$,

$$
p_{k}(X)=p_{k}\left(-\widetilde{\nu}_{X}\right) \in H^{4 k}(X ; \mathbb{Q}) \quad(k \geq 0) .
$$

(ii) The $\mathcal{L}$-genus of $X$ is the $\mathcal{L}$-genus of $-\widetilde{\nu}_{X}$,

$$
\mathcal{L}(X)=\mathcal{L}\left(-\widetilde{\nu}_{X}\right) \in H^{4 *}(X ; \mathbb{Q}) .
$$

(iii) The $\mathcal{L}^{H}$-genus of $X$ is

$$
\mathcal{L}^{H}(X)=8 i(X)+\mathcal{L}(X) \in H^{4 *}(X ; \mathbb{Q})
$$

with components

$$
\mathcal{L}_{k}^{H}(X)= \begin{cases}1+8 i(X) \in H^{0}(X ; \mathbb{Q}) & \text { if } k=0 \\ \mathcal{L}_{k}(X) \in H^{4 k}(X ; \mathbb{Q}) & \text { if } k \geq 1 .\end{cases}
$$

The $\mathcal{L}$-genus has the same expression in terms of the Pontrjagin classes of a homology manifold $X$ as for a topological manifold, with components

$$
\begin{aligned}
& \mathcal{L}_{0}(X)=p_{0}(X)=1 \in H^{0}(X ; \mathbb{Q}) \\
& \mathcal{L}_{1}(X)=\frac{1}{3} p_{1}(X) \in H^{4}(X ; \mathbb{Q}) \\
& \mathcal{L}_{2}(X)=\frac{1}{45}\left(7 p_{2}(X)-p_{1}(X)^{2}\right) \in H^{8}(X ; \mathbb{Q}), \quad \text { etc. }
\end{aligned}
$$

The Hirzebruch signature theorem also applies to homology manifolds: 
Corollary 7.4. (i) If $X$ is an m-dimensional homology manifold and $f: M=$ $X_{T O P} \rightarrow X$ is the canonical normal map, then

$$
\mathcal{L}(M)=f^{*} \mathcal{L}(X) \in H^{4 *}(M ; \mathbb{Q}) .
$$

(ii) The canonical $\mathbb{L}^{\bullet}$-orientation of an $m$-dimensional homology manifold $X$ is given rationally by (the Poincaré dual of) the $\mathcal{L}^{H}$-genus

$$
[X]_{\mathbb{L}} \otimes 1=\mathcal{L}^{H}(X) \in H_{m}\left(X ; \mathbb{L}^{\bullet}\right) \otimes \mathbb{Q}=H_{m-4 *}(X ; \mathbb{Q})=H^{4 *}(X ; \mathbb{Q}) .
$$

(iii) The signature of a $4 k$-dimensional homology manifold $X$ is given by

$$
\sigma^{*}(X)=\left\langle\mathcal{L}_{k}(X),[X]\right\rangle \in L^{4 k}(\mathbb{Z})=\mathbb{Z} .
$$

Proof. (i) is immediate from $f^{*}\left(-\widetilde{\nu}_{X}\right)=\tau_{M}$ (stably).

(ii) The canonical $\mathbb{L} \bullet$-orientation of a topological manifold $M$ is given rationally by the ordinary $\mathcal{L}$-genus

$$
[M]_{\mathbb{L}} \otimes 1=\mathcal{L}(M) \in H_{m}\left(M ; \mathbb{L}^{\bullet}\right) \otimes \mathbb{Q}=H_{m-4 *}(M ; \mathbb{Q})=H^{4 *}(M ; \mathbb{Q}),
$$

with $\mathcal{L}^{H}(M)=\mathcal{L}(M), i(M)=0$. If $f: M=X_{T O P} \rightarrow X$ is the canonical normal map, then

$$
\begin{aligned}
{[X]_{\mathbb{L}} \otimes 1 } & =f_{*}[M]_{\mathbb{L}} \otimes 1+8 i(X) \\
& =f_{*} \mathcal{L}(M)+8 i(X)=\mathcal{L}(X)+8 i(X)=\mathcal{L}^{H}(X) \in H^{4 *}(M ; \mathbb{Q}) .
\end{aligned}
$$

(iii) is immediate from (ii), the identity $\sigma^{*}(X)=A\left([X]_{\mathbb{L}}\right)$ of Theorem 7.1] and the fact that the simply-connected assembly map

$$
\begin{aligned}
A: H_{4 k}\left(X ; \mathbb{L}^{\bullet}\right) & =H_{4 k}\left(X ; L^{0}(\mathbb{Z})\right) \oplus H_{4 k}(X ; \mathbb{L} \bullet\langle 1\rangle) \\
& \stackrel{\left(0 p_{*}\right)}{\longrightarrow} H_{4 k}(\{*\} ; \mathbb{L} \bullet\langle 1\rangle)=L^{4 k}(\mathbb{Z})
\end{aligned}
$$

sends $8 i(X) \in H_{4 k}\left(X ; L^{0}(\mathbb{Z})\right)$ to 0 , with $p: X \rightarrow\{*\}$ the unique map.

Corollary 7.5. Let $X=M_{i}$ be the $m$-dimensional homology manifold with prescribed resolution obstruction $i(X)=i$ given by the construction of 1.5 applied to an m-dimensional topological manifold $M(m \geq 6)$, with normal map $f: X \rightarrow M$.

(i) The canonical $\mathbb{L}^{\bullet}$-orientation $[X]_{\mathbb{L}} \in H_{m}\left(X ; \mathbb{L}^{\bullet}\right)$ is such that

$$
f_{*}[X]_{\mathbb{L}}=8 i+[M]_{\mathbb{L}} \in H_{m}\left(M ; \mathbb{L}^{\bullet}\right) .
$$

(ii) The $\mathcal{L}^{H}$-genus $\mathcal{L}^{H}(X) \in H^{4 *}(X ; \mathbb{Q})=H_{m-4 *}(X ; \mathbb{Q})$ is such that

$$
f_{*} \mathcal{L}^{H}(X)=8 i+\mathcal{L}(M) \in H^{4 *}(M ; \mathbb{Q})
$$

Proof. (i) For any normal map $F: X \rightarrow Y$ of $m$-dimensional homology manifolds we have

$$
F_{*}[X]_{\mathbb{L}}=(1+T)[F]_{\mathbb{L}}+[Y]_{\mathbb{L}} \in H_{m}\left(Y ; \mathbb{L}^{\bullet}\right) .
$$

For $F=f: X \rightarrow Y=M$

$$
[F]_{\mathbb{L}}=i \in H_{m}\left(M ; \overline{\mathbb{L}}_{\bullet}\right)
$$

and $(1+T)[F]_{\mathbb{L}}=8 i$.

(ii) is immediate from (i) and 7.4 . 
We shall now analyze the difference

$$
\widetilde{\nu}_{X \times Y}-\widetilde{\nu}_{X} \times \widetilde{\nu}_{Y}: X \times Y \rightarrow G / T O P
$$

for homology manifolds $X, Y$ using the canonical $\mathbb{L} \bullet$-orientation of homology manifolds and the surgery composition and product formulae:

(i) (Ranicki [14 (4.3)) The composite of normal maps $f: X \rightarrow Y, g: Y \rightarrow Z$ of $n$-dimensional Poincaré spaces is a normal map $g f: X \rightarrow Z$ with surgery obstruction

$$
\sigma_{*}(g f)=\sigma_{*}(f)+\sigma_{*}(g) \in L_{n}\left(\mathbb{Z}\left[\pi_{1}(Z)\right]\right) .
$$

(ii) (14 (8.1)) The product of a normal map $f: M \rightarrow X$ of $m$-dimensional Poincaré spaces and a normal map $g: N \rightarrow Y$ of $n$-dimensional Poincaré spaces is a normal map $f \times g: M \times N \rightarrow X \times Y$ of $(m+n)$-dimensional geometric Poincaré spaces with surgery obstruction

$$
\begin{aligned}
\sigma_{*}(f \times g) & =\sigma_{*}(f) \otimes \sigma_{*}(g)+\sigma^{*}(X) \otimes \sigma_{*}(g)+\sigma_{*}(f) \otimes \sigma^{*}(Y) \\
& =\sigma^{*}(M) \otimes \sigma_{*}(g)+\sigma_{*}(f) \otimes \sigma^{*}(Y) \in L_{m+n}\left(\mathbb{Z}\left[\pi_{1}(X \times Y)\right]\right) .
\end{aligned}
$$

The formulae are proved on the chain level, using the Eilenberg-Zilber theorem.

Theorem 7.6. Let $X$ be an $m$-dimensional homology manifold, and let $Y$ be an $n$-dimensional homology manifold.

(i) The product $(m+n)$-dimensional homology manifold $X \times Y$ has canonical $\mathbb{L} \bullet$-orientation the product of the canonical $\mathbb{L} \bullet$-orientations of $X$ and $Y$ :

$$
[X \times Y]_{\mathbb{L}}=[X]_{\mathbb{L}} \otimes[Y]_{\mathbb{L}} \in H_{m+n}\left(X \times Y ; \mathbb{L}^{\bullet}\right) .
$$

(ii) Let $f: X_{T O P} \rightarrow X, g: Y_{T O P} \rightarrow Y$ be normal maps from topological manifolds in the normal bordism classes determined by the canonical TOP reductions $\widetilde{\nu}_{X}, \widetilde{\nu}_{Y}$ of $\nu_{X}, \nu_{Y}$, and let

$$
h:(X \times Y)_{T O P} \rightarrow X_{T O P} \times Y_{T O P}
$$

be the normal map of topological manifolds classified by

$$
\begin{aligned}
{[h]_{\mathbb{L}} } & \left.=i(X) \otimes \widetilde{Y_{T O P}}\right]_{\mathbb{L}}+\left[\widetilde{X_{T O P}}\right]_{\mathbb{L}} \otimes i(Y) \\
& \in H_{m+n}\left(X_{T O P} \times Y_{T O P} ; \mathbb{L}_{\bullet}\right)=\left[X_{T O P} \times Y_{T O P}, G / T O P\right] .
\end{aligned}
$$

The composite normal map

$$
(X \times Y)_{T O P} \stackrel{h}{\longrightarrow} X_{T O P} \times Y_{T O P} \stackrel{f \times g}{\longrightarrow} X \times Y
$$

is in the normal bordism class determined by the canonical TOP reduction $\widetilde{\nu}_{X \times Y}$ of $\nu_{X \times Y}$.

(iii) The canonical TOP reduction

$$
\widetilde{\nu}_{X \times Y}: X \times Y \rightarrow B T O P
$$

of the Spivak normal fibration of $X \times Y$

$$
\nu_{X \times Y}=\nu_{X} \times \nu_{Y}: X \times Y \rightarrow B G
$$

differs from the product TOP reduction

$$
\widetilde{\nu}_{X} \times \widetilde{\nu}_{Y}: X \times Y \rightarrow B T O P
$$


by the element

$$
\begin{aligned}
t\left(\widetilde{\nu}_{X \times Y}, \widetilde{\nu}_{X} \times \widetilde{\nu}_{Y}\right) & =i(X) \otimes[\widetilde{[Y}]_{\mathbb{L}}+\widetilde{[X}_{\mathbb{L}} \otimes i(Y) \\
& \in H_{m+n}\left(X \times Y ; \mathbb{L}_{\bullet}\right)=[X \times Y, G / T O P] .
\end{aligned}
$$

Proof. (i) This is just the Eilenberg-Zilber theorem on the level of symmetric Poincaré cycles.

(ii) By construction, $f$ and $g$ are classified by

$$
[f]_{\mathbb{L}}=-i(X) \in H_{m}\left(X ; \overline{\mathbb{L}}_{\bullet}\right), \quad[g]_{\mathbb{L}}=-i(Y) \in H_{n}\left(Y ; \overline{\mathbb{L}}_{\bullet}\right) .
$$

By the surgery product formula (on the level of quadratic Poincaré cycles) the product normal map $f \times g: X_{T O P} \times Y_{T O P} \rightarrow X \times Y$ is classified by

$$
\begin{aligned}
{[f \times g]_{\mathbb{L}} } & =[X]_{\mathbb{L}} \otimes[g]_{\mathbb{L}}+[f]_{\mathbb{L}} \otimes g_{*}\left[Y_{T O P}\right]_{\mathbb{L}} \\
& \left.=-[X]_{\mathbb{L}} \otimes i(Y)-i(X) \otimes(1+\widetilde{[Y}]_{\mathbb{L}}\right) \in H_{m+n}\left(X \times Y ; \overline{\mathbb{L}}_{\bullet}\right) .
\end{aligned}
$$

By the surgery composition formula (on the level of quadratic Poincaré cycles),

$$
\begin{aligned}
& {[(f \times g) h]_{\mathbb{L}}=(f \times g)_{*}[h]_{\mathbb{L}}+[f \times g]_{\mathbb{L}}} \\
& =i(X) \otimes[\widetilde{[Y}]_{\mathbb{L}}+\widetilde{[X]_{\mathbb{L}}} \otimes i(Y) \\
& -[X]_{\mathbb{L}} \otimes i(Y)-i(X) \otimes g_{*}\left[Y_{T O P}\right]_{\mathbb{L}} \\
& =-i(X) * i(Y)=-i(X \times Y) \\
& \in H_{m+n}\left(X \times Y ; L_{0}(\mathbb{Z})\right) \subseteq H_{m+n}\left(X \times Y ; \overline{\mathbb{L}}_{\bullet}\right),
\end{aligned}
$$

so that $(f \times g) h$ is in the canonical normal bordism class.

(iii) is immediate from (ii), noting that

$$
\begin{aligned}
{[h]_{\mathbb{L}} } & =\left(t\left(\widetilde{\nu}_{X \times Y}, \widetilde{\nu}_{X} \times \widetilde{\nu}_{Y}\right), 0\right) \\
& \in H_{m+n}\left(X_{T O P} \times Y_{T O P} ; \mathbb{L}_{\bullet}\right)=H_{m+n}\left(X \times Y ; \mathbb{L}_{\bullet}\right) \oplus K_{m+n}
\end{aligned}
$$

with $K_{m+n}$ the $(m+n)$-dimensional homology kernel of $f \times g$.

Definition 7.7. For any integers $i, j \in \mathbb{Z}$ let

$$
i * j=i+j+8 i j \in \mathbb{Z},
$$

so that

$$
(1+8 i)(1+8 j)=1+8 i * j \in \mathbb{Z} .
$$

Corollary 7.8. Let $X, Y$ be homology manifolds.

(i) The $\mathcal{L}^{H}$-genus of $X \times Y$ is

$$
\mathcal{L}^{H}(X \times Y)=\mathcal{L}^{H}(X) \otimes \mathcal{L}^{H}(Y) \in H^{4 *}(X \times Y ; \mathbb{Q}),
$$

with

$$
\begin{aligned}
\mathcal{L}_{k}^{H}(X \times Y)= & \sum_{\substack{i+j=k\\
}}\left(\mathcal{L}_{i}(X) \otimes \mathcal{L}_{j}(Y)\right)+\mathcal{L}_{k}(X) \otimes 8 i(Y)+8 i(X) \otimes \mathcal{L}_{k}(Y) \\
& =H^{4 k}(X)(k \geq 1) .
\end{aligned}
$$

(ii) (Quinn [12]) The resolution obstruction of $X \times Y$ is

$$
i(X \times Y)=i(X) * i(Y) \in \mathbb{Z} .
$$

Proof. (i) This is just the rationalization of the product formula of Theorem [7.6 (i).

(ii) This is just the 0-dimensional component of the identity of (i). 
As is well-known, the $\mathcal{L}$-genus determines the Pontrjagin classes, so the formula in Corollary 7.8 can be used to determine the Pontrjagin classes of a product homology manifold $X \times Y$ :

$$
p_{k}(X \times Y) \in H^{4 k}(X \times Y ; \mathbb{Q}) \quad(k \geq 1)
$$

in terms of the Pontrjagin classes $p_{*}(X), p_{*}(Y)$ and the resolution obstructions $i(X), i(Y)$.

Example 7.9. If $X, Y$ are 4-dimensional homology manifolds, then the Pontrjagin classes of the product 8-dimensional homology manifold $X \times Y$ are given by

$$
\begin{aligned}
& p_{1}(X \times Y)=p_{1}(X) \otimes(1+8 i(Y))+(1+8 i(X)) \otimes p_{1}(Y) \in H^{4}(X \times Y ; \mathbb{Q}), \\
& p_{2}(X \times Y)=\left(1+\frac{16}{7} i(X) * i(Y)\right) p_{1}(X) \otimes p_{1}(Y) \in H^{8}(X \times Y ; \mathbb{Q})
\end{aligned}
$$

Corollary 7.10. The homology manifold bordism product for $m \geq 6, n \geq 6$

$$
\Omega_{m}^{H}(J) \otimes \Omega_{n}^{H}(K) \rightarrow \Omega_{m+n}^{H}(J \times K) ; X \otimes Y \mapsto X \times Y
$$

corresponds under the isomorphisms of $[1.6$ to the topological manifold bordism product

$$
\begin{gathered}
\Omega_{m}^{T O P}(J)\left[L_{0}(\mathbb{Z})\right] \otimes \Omega_{n}^{T O P}(K)\left[L_{0}(\mathbb{Z})\right] \rightarrow \Omega_{m+n}^{T O P}(J \times K)\left[L_{0}(\mathbb{Z})\right] \\
M[i] \otimes N[j] \mapsto\left(M_{i} \times N_{j}\right)_{T O P}[i * j] .
\end{gathered}
$$

Here, $i=I(X), j=I(Y)$, and $M=X_{T O P} \rightarrow X, N=Y_{T O P} \rightarrow Y$ are the normal maps from topological manifolds determined by the canonical TOP reductions of $\nu_{X}, \nu_{Y}$, and $M_{i} \rightarrow M, N_{j} \rightarrow N$ are the normal maps from homology manifolds with resolution obstructions $I\left(M_{i}\right)=i, I\left(N_{j}\right)=j$ given by Proposition 1.5 . The normal map $h:(X \times Y)_{T O P} \rightarrow M \times N$ of Theorem 7.6 (ii) classified by

$$
[h]_{\mathbb{L}}=i \otimes \widetilde{[N]_{\mathbb{L}}}+\widetilde{[M]_{\mathbb{L}}} \otimes j \in H_{m+n}\left(M \times N ; \mathbb{L}_{\bullet}\right)
$$

is bordant to the composite normal map

$$
F:\left(M_{i} \times N_{j}\right)_{T O P} \rightarrow M_{i} \times N_{j} \rightarrow M \times N .
$$

Proof. The normal maps $M_{i} \rightarrow M, N_{j} \rightarrow N$ are classified by

$$
\left[M_{i} \rightarrow M\right]_{\mathbb{L}}=i \in H_{m}\left(M ; \overline{\mathbb{L}}_{\bullet}\right),\left[N_{j} \rightarrow N\right]_{\mathbb{L}}=j \in H_{n}\left(N ; \overline{\mathbb{L}}_{\bullet}\right) .
$$

Apply the surgery composition and product formulae to compute the classifying invariant of the composite $\left(M_{i} \times N_{j}\right)_{T O P} \rightarrow M_{i} \times N_{j} \rightarrow M \times N$ :

$$
\begin{aligned}
{\left[\left(M_{i}\right.\right.} & \left.\left.\times N_{j}\right)_{T O P} \rightarrow M \times N\right]_{\mathbb{L}} \\
& =\left[\left(M_{i} \times N_{j}\right)_{T O P} \rightarrow M_{i} \times N_{j}\right]+\left[M_{i} \times N_{j} \rightarrow M \times N\right] \\
& =-i * j+8 i j+i \otimes[N]_{\mathbb{L}}+[M]_{\mathbb{L}} \otimes j \\
& =i \otimes \widetilde{[N]_{\mathbb{L}}}+\widetilde{[M]_{\mathbb{L}}} \otimes j \in H_{m+n}\left(M \times N ; \mathbb{L}_{\bullet}\right) \subseteq H_{m+n}\left(M \times N ; \overline{\mathbb{L}}_{\bullet}\right),
\end{aligned}
$$

so that $\left(M_{i} \times N_{j}\right)_{T O P} \rightarrow M \times N,(X \times Y)_{T O P} \rightarrow M \times N$ are normal bordant. 
Remark 7.11. Given an $m$-dimensional topological manifold $M$, an $n$-dimensional topological manifold $N$, and $i, j \in \mathbb{Z}$, let

$$
P=\left(M_{i} \times N_{j}\right)_{T O P}
$$

be the $(m+n)$-dimensional topological manifold appearing in Corollary 7.10, and let $F: P \rightarrow M \times N$ be the degree 1 normal map. The $\mathcal{L}$-genus of $P$ has components

$$
\begin{aligned}
\mathcal{L}_{k}(P) & =F^{*}\left((\mathcal{L}(M) \otimes \mathcal{L}(N))_{k}+8 i \otimes \mathcal{L}_{k}(N)+\mathcal{L}_{k}(M) \otimes 8 j\right) \\
& \in H^{4 k}(P ; \mathbb{Q}) \quad(k \geq 1)
\end{aligned}
$$

Example 7.12. Take $m=n=4, J=K=\{$ pt. $\}$ in 7.10, The signature defines an isomorphism

$$
\omega_{4}: \Omega_{4}^{T O P} \rightarrow \mathbb{Z} ; M \mapsto \frac{1}{3}\left\langle p_{1}(M),[M]\right\rangle=\sigma^{*}(M)
$$

such that

$$
\omega_{4}\left(\mathbb{C} \mathbb{P}^{2}\right)=1
$$

Linear combinations of Pontrjagin numbers define an isomorphism

$$
\begin{aligned}
\omega_{8}: & \Omega_{8}^{T O P} \otimes \mathbb{Q} \rightarrow \mathbb{Q} \oplus \mathbb{Q} ; \\
& P \mapsto\left(\frac{1}{9}\left\langle 5 p_{2}(P)-2 p_{1}(P)^{2},[P]\right\rangle, \frac{1}{5}\left\langle-2 p_{2}(P)+p_{1}(P)^{2},[P]\right\rangle\right)
\end{aligned}
$$

such that

$$
\omega_{8}\left(\mathbb{C} \mathbb{P}^{2} \times \mathbb{C} \mathbb{P}^{2}\right)=(1,0), \omega_{8}\left(\mathbb{C} \mathbb{P}^{4}\right)=(0,1),
$$

with the signature given by

$$
\begin{aligned}
\sigma^{*}: \Omega_{8}^{T O P} & \otimes \mathbb{Q} \stackrel{\omega_{8}}{\rightarrow} \mathbb{Q} \oplus \mathbb{Q} \stackrel{(11)}{\rightarrow} L^{8}(\mathbb{Z}) \otimes \mathbb{Q}=\mathbb{Q} ; \\
P \mapsto \sigma^{*}(P) & =\frac{1}{9}\left\langle 5 p_{2}(P)-2 p_{1}(P)^{2},[P]\right\rangle+\frac{1}{5}\left\langle-2 p_{2}(P)+p_{1}(P)^{2},[P]\right\rangle \\
& =\frac{1}{45}\left\langle 7 p_{2}(P)-p_{1}(P)^{2},[P]\right\rangle .
\end{aligned}
$$

If $M, N$ are 4-dimensional topological manifolds, then

$$
\omega_{8}(M \times N)=\left(\sigma^{*}(M) \sigma^{*}(N), 0\right)
$$

and if $P=\left(M_{i} \times N_{j}\right)_{T O P}$ for some $i, j \in \mathbb{Z}$, then

$$
\begin{aligned}
& \left\langle p_{1}(P)^{2},[P]\right\rangle=2(1+8 i * j)\left\langle p_{1}(M),[M]\right\rangle\left\langle p_{1}(N),[N]\right\rangle \\
& \left\langle p_{2}(P),[P]\right\rangle=\left(1+\frac{16}{7} i * j\right)\left\langle p_{1}(M),[M]\right\rangle\left\langle p_{1}(N),[N]\right\rangle \\
& \omega_{8}(P)=\left(\left(1-\frac{144}{7} i * j\right) \sigma^{*}(M) \sigma^{*}(N),\left(\frac{144}{7} i * j\right) \sigma^{*}(M) \sigma^{*}(N)\right) .
\end{aligned}
$$

The product structure on homology manifold bordism

$$
\Omega_{m}^{H} \otimes \Omega_{n}^{H} \rightarrow \Omega_{m+n}^{H} ; X \otimes Y \mapsto X \times Y
$$

therefore does not correspond to the product structure

$$
\Omega_{m}^{T O P}\left[L_{0}(\mathbb{Z})\right] \otimes \Omega_{n}^{T O P}\left[L_{0}(\mathbb{Z})\right] \rightarrow \Omega_{m+n}^{T O P}\left[L_{0}(\mathbb{Z})\right] ; M[i] \otimes N[j] \mapsto(M \times N)[i * j]
$$


under the abelian group isomorphism of 1.6

$$
\Omega_{*}^{H} \cong \Omega_{*}^{T O P}\left[L_{0}(\mathbb{Z})\right] .
$$

However, as stated in Weinberger [19], for $m \geq 6$ there is an isomorphism of rings

$$
\phi: \Omega_{*}^{H} \otimes \mathbb{Q} \cong\left(\Omega_{*}^{T O P} \otimes \mathbb{Q}\right)\left[L_{0}(\mathbb{Z})\right] .
$$

We shall now give an explicit description of the isomorphism $\phi$, which is different from the isomorphism $\Omega_{m}^{H} \cong \Omega_{m}^{T O P}\left[L_{0}(\mathbb{Z})\right]$ given by 1.6

Recall that the rational cobordism groups

$$
\Omega_{*}^{T O P} \otimes \mathbb{Q}=\Omega_{*}^{O} \otimes \mathbb{Q}
$$

are detected by Pontrjagin numbers in dimensions $* \equiv 0(\bmod 4)$, and are 0 in dimensions $* \not \equiv 0(\bmod 4)$. The Pontrjagin numbers of an $m$-dimensional topological manifold $M$ with $m \equiv 0(\bmod 4)$ are rational linear combinations of the characteristic $\mathcal{L}$-numbers

$$
\mathcal{L}_{I}(M)=\left\langle\mathcal{L}_{i_{1}}(M) \mathcal{L}_{i_{2}}(M) \cdots \mathcal{L}_{i_{k}}(M),[M]\right\rangle \in \mathbb{Q},
$$

one for each $k$-tuple $I=\left(i_{1}, i_{2}, \ldots, i_{k}\right)$ of integers $\geq 1$ with $4\left(i_{1}+i_{2}+\cdots+i_{k}\right)=m$. Conversely, the characteristic $\mathcal{L}$-numbers of $M$ are rational linear combinations of the Pontrjagin numbers. Thus the characteristic $\mathcal{L}$-numbers also determine the rational cobordism class, with isomorphisms

$$
\Omega_{m}^{T O P} \otimes \mathbb{Q} \rightarrow \sum_{I} \mathbb{Q} ; M \mapsto \sum_{I} \mathcal{L}_{I}(M) .
$$

Definition 7.13. (i) The $\mathcal{L}^{\prime}$-genus of an $m$-dimensional homology manifold $X$ is

$$
\mathcal{L}^{\prime}(X)=\frac{1}{1+8 i(X)} \mathcal{L}^{H}(X) \in H^{4 *}(X ; \mathbb{Q}),
$$

with components

$$
\mathcal{L}_{k}^{\prime}(X)= \begin{cases}1 \in H^{0}(X ; \mathbb{Q}) & \text { if } k=0 \\ \frac{1}{1+8 i(X)} \mathcal{L}_{k}(X) \in H^{4 k}(X ; \mathbb{Q}) & \text { if } k \geq 1 .\end{cases}
$$

(ii) The $\mathcal{L}^{\prime}$-characteristic numbers of an $m$-dimensional homology manifold $X$ are

$$
\begin{gathered}
\mathcal{L}_{I}^{\prime}(X)=\left\langle\mathcal{L}_{i_{1}}^{\prime}(X) \mathcal{L}_{i_{2}}^{\prime}(X) \ldots \mathcal{L}_{i_{k}}^{\prime}(X),[X]\right\rangle \in \mathbb{Q} \\
\left(I=\left(i_{1}, i_{2}, \ldots, i_{k}\right), m=4\left(i_{1}+i_{2}+\cdots+i_{k}\right)\right)
\end{gathered}
$$

with $\mathcal{L}_{I}^{\prime}(X)=0$ if $m \not \equiv 0(\bmod 4)$.

(iii) Define a function

$$
\phi: \Omega_{m}^{H} \otimes \mathbb{Q} \rightarrow\left(\Omega_{m}^{T O P} \otimes \mathbb{Q}\right)\left[L_{0}(\mathbb{Z})\right] ; X \mapsto(M \otimes a)[i(X)]
$$

using any $m$-dimensional topological manifold $M$ and $a \in \mathbb{Q}$ such that

$$
\mathcal{L}_{I}^{\prime}(X)=\mathcal{L}_{I}(M) \otimes a \in \mathbb{Q}
$$

for every $I=\left(i_{1}, i_{2}, \ldots, i_{k}\right)$ with $4\left(i_{1}+i_{2}+\cdots+i_{k}\right)=m$.

The morphism of 1.6

$$
\psi: \Omega_{m}^{H} \rightarrow \Omega_{m}^{T O P}\left[L_{0}(\mathbb{Z})\right] ; X \mapsto X_{T O P}[i(X)]
$$


(which is an isomorphism for $m \geq 6$ ) is such that the composite

$$
\Omega_{m}^{H} \otimes \mathbb{Q} \stackrel{\psi}{\rightarrow}\left(\Omega_{m}^{T O P} \otimes \mathbb{Q}\right)\left[L_{0}(\mathbb{Z})\right] \cong\left(\sum_{I} \mathbb{Q}\right)\left[L_{0}(\mathbb{Z})\right]
$$

sends an $m$-dimensional homology manifold $X$ to

$$
\left(\sum_{I} \mathcal{L}_{I}(X)\right)[i(X)] \in\left(\sum_{I} \mathbb{Q}\right)\left[L_{0}(\mathbb{Z})\right]
$$

The composite

$$
\Omega_{m}^{H} \otimes \mathbb{Q} \stackrel{\phi}{\rightarrow}\left(\Omega_{m}^{T O P} \otimes \mathbb{Q}\right)\left[L_{0}(\mathbb{Z})\right] \cong\left(\sum_{I} \mathbb{Q}\right)\left[L_{0}(\mathbb{Z})\right]
$$

sends an $m$-dimensional homology manifold $X$ to

$$
\left(\sum_{I} \mathcal{L}_{I}(M) \otimes a\right)[i(X)]=\left(\sum_{I} \mathcal{L}_{I}^{\prime}(X)\right)[i(X)] \in\left(\sum_{I} \mathbb{Q}\right)\left[L_{0}(\mathbb{Z})\right],
$$

so that $\phi \neq \psi$. The inverse of $\psi$ for $m \geq 6$ is given by

$$
\psi^{-1}: \Omega_{m}^{T O P}\left[L_{0}(\mathbb{Z})\right] \rightarrow \Omega_{m}^{H} ; N[i] \mapsto N_{i}
$$

with $N_{i}$ the homology manifold with resolution obstruction $i$ obtained by the construction of 1.5 . The composite

$$
\left(\Omega_{m}^{T O P} \otimes \mathbb{Q}\right)\left[L_{0}(\mathbb{Z})\right] \stackrel{\psi^{-1}}{\rightarrow} \Omega_{m}^{H} \otimes \mathbb{Q} \stackrel{\phi}{\rightarrow}\left(\Omega_{m}^{T O P} \otimes \mathbb{Q}\right)\left[L_{0}(\mathbb{Z})\right] \cong\left(\sum_{I} \mathbb{Q}\right)\left[L_{0}(\mathbb{Z})\right]
$$

is given by

$$
N[i] \mapsto\left(\sum_{I}\left(\frac{1}{1+8 i}\right)^{k} \mathcal{L}_{I}\left(N_{i}\right)\right)[i]=\left(\sum_{I}\left(\frac{1}{1+8 i}\right)^{k} \mathcal{L}_{I}(N)\right)[i]
$$

with $I=\left(i_{1}, i_{2}, \ldots, i_{k}\right), m=4\left(i_{1}+i_{2}+\cdots+i_{k}\right)$.

Proposition 7.14. (i) The function $\phi: \Omega_{m}^{H} \otimes \mathbb{Q} \rightarrow\left(\Omega_{m}^{T O P} \otimes \mathbb{Q}\right)\left[L_{0}(\mathbb{Z})\right]$ is a ring morphism which is an isomorphism for $m \geq 6$.

(ii) The rational homology manifold bordism product

$$
\left(\Omega_{m}^{H} \otimes \mathbb{Q}\right) \otimes\left(\Omega_{n}^{H} \otimes \mathbb{Q}\right) \rightarrow \Omega_{m+n}^{H} \otimes \mathbb{Q} ;(X \otimes Y) \mapsto(X \times Y)
$$

corresponds under $\phi$ to the rational topological manifold bordism product

$$
\begin{gathered}
\left(\Omega_{m}^{T O P} \otimes \mathbb{Q}\right)\left[L_{0}(\mathbb{Z})\right] \otimes\left(\Omega_{n}^{T O P} \otimes \mathbb{Q}\right)\left[L_{0}(\mathbb{Z})\right] \rightarrow\left(\Omega_{m+n}^{T O P} \otimes \mathbb{Q}\right)\left[L_{0}(\mathbb{Z})\right], \\
(M \otimes a)[i] \otimes(N \otimes b)[j] \mapsto((M \times N) \otimes a b)[i * j] .
\end{gathered}
$$

Here $i=i(X), j=i(Y)$, and $M \otimes a=\phi(X), N \otimes b=\phi(Y)$.

Proof. We know from 1.6 that the morphism of abelian groups

$\psi: \Omega_{m}^{H} \otimes \mathbb{Q} \rightarrow\left(\Omega_{m}^{T O P} \otimes \mathbb{Q}\right)\left[L_{0}(\mathbb{Z})\right] \cong\left(\sum_{I} \mathbb{Q}\right)\left[L_{0}(\mathbb{Z})\right] ; X \mapsto\left(\sum_{I} \mathcal{L}_{I}(X)\right)[i(X)]$

is an isomorphism for $m \geq 6$. It follows that the morphism of abelian groups

$$
\phi: \Omega_{m}^{H} \otimes \mathbb{Q} \rightarrow\left(\Omega_{m}^{T O P} \otimes \mathbb{Q}\right)\left[L_{0}(\mathbb{Z})\right] \cong\left(\sum_{I} \mathbb{Q}\right)\left[L_{0}(\mathbb{Z})\right] ; X \mapsto\left(\sum_{I} \mathcal{L}_{I}^{\prime}(X)\right)[i(X)]
$$

is also an isomorphism for $m \geq 6$. It remains to show that $\phi$ preserves the multiplicative structures. By 7.8 (ii),

$$
\phi(X \times Y)=(P \otimes c)[i * j]
$$


for some element $P \otimes c \in \Omega_{m+n}^{T O P} \otimes \mathbb{Q}$. We need to show that

$$
P \otimes c=(M \times N) \otimes a b \in \Omega_{m+n}^{T O P} \otimes \mathbb{Q} .
$$

From the above we see that $M \otimes a$ and $N \otimes b$ correspond to the $\mathcal{L}$-numbers $\mathcal{L}_{I}(M \otimes a)=\mathcal{L}_{I}^{\prime}(X)$ and $\mathcal{L}_{I}(N \otimes b)=\mathcal{L}_{I}^{\prime}(Y) \in \mathbb{Q}$ respectively. We compare these $\mathcal{L}$-numbers with those of $P \otimes c$, which are given by

$$
\mathcal{L}_{I}(P \otimes c)=\mathcal{L}_{I}^{\prime}(X \times Y) \in \mathbb{Q} .
$$

We observe that by 7.8 (i)

$$
\begin{aligned}
\mathcal{L}^{\prime}(X \times Y) & =\frac{1}{1+8 i * j} \mathcal{L}^{H}(X \times Y) \\
& =\frac{1}{1+8 i * j} \mathcal{L}^{H}(X) \otimes \mathcal{L}^{H}(Y) \\
& =\mathcal{L}^{\prime}(X) \otimes \mathcal{L}^{\prime}(Y) \in H^{4 *}(X \times Y ; \mathbb{Q})
\end{aligned}
$$

and that the $\mathcal{L}$-numbers satisfy the following product formula:

$$
\mathcal{L}_{I}((M \times N) \otimes a b)=\sum_{J, K} \mathcal{L}_{J}(M \otimes a) \mathcal{L}_{K}(N \otimes b) \in \mathbb{Q},
$$

with $I=\left(i_{1}, i_{2}, \ldots, i_{l}\right)$, and $J=\left(j_{1}, j_{2}, \ldots, j_{l}\right), K=\left(k_{1}, k_{2}, \ldots, k_{l}\right)$ such that

$$
j_{1}+k_{1}=i_{1}, j_{2}+k_{2}=i_{2}, \ldots, j_{l}+k_{l}=i_{l} .
$$

Conversely,

$$
\begin{aligned}
\sum_{J, K} \mathcal{L}_{J}(M \otimes a) \mathcal{L}_{K}(N \otimes b)=\sum_{J, K} \mathcal{L}_{J}^{\prime}(X) \mathcal{L}_{K}^{\prime}(Y) \\
=\sum_{J, K} \mathcal{L}_{J}^{\prime}(X) \otimes \mathcal{L}_{K}^{\prime}(Y)=\left(\mathcal{L}^{\prime}(X) \otimes \mathcal{L}^{\prime}(Y)\right)_{I} \\
=\mathcal{L}_{I}^{\prime}(X \times Y)=\mathcal{L}_{I}(P \otimes c) \in \mathbb{Q}
\end{aligned}
$$

\section{REFERENCES}

1. J. Bryant, S. Ferry, W. Mio, S. Weinberger, Topology of homology manifolds, Ann. of Math. 143, 435-467 (1996). MR 97b:57017

2. T.A. Chapman, Simple homotopy theory for ANR's, General Topology and its Applications 7, 165-174 (1977). MR 58:18414

3. M. Cohen, Simplicial structures and transverse cellularity, Ann. of Math. 85, 218-245 (1967). MR 35:1037

4. S. Ferry, E.K. Pedersen, Epsilon surgery theory I, In: Novikov Conjectures, Index Theorems and Rigidity, Vol. 2 (Oberwolfach, 1993), Lond. Math. Soc. Lecture Note Ser. 227, Cambridge University Press (1995), 167-226. MR 97g:57044

5. B. Hughes, L. Taylor, B. Williams, Manifold approximate fibrations are approximately bundles, Forum Math. 3, 309-325 (1991). MR 92k:57040

6. H. Johnston, Transversality for homology manifolds, Topology 38, 673-697 (1999). MR 99k:57048

7. L. Jones, Patch spaces: a geometric representation for Poincaré spaces, Ann. of Math. 97, 306-343 (1973); 102, 183-185 (1975) MR 47:4269 |MR 52:11930

8. R. Kirby, L. Siebenmann, Foundational essays on topological manifolds, smoothings, and triangulations, Ann. of Math. Study 88, Princeton University Press (1977). MR 58:31082

9. N. Levitt, A. Ranicki, Intrinsic transversality structures, Pacific J. Math. 129, 85-144 (1987). MR 88m:57027

10. A. Marin, La transversalité topologique, Ann. of Math. 106, 269-293 (1977). MR 57:10707 
11. F. Quinn, Resolutions of homology manifolds, and the topological characterization of manifolds, Invent. Math. 72, 264-284 (1983); Corrigendum 85 (1986) 653. MR 85b:57023: MR 87g:57031

12. - An obstruction to the resolution of homology manifolds, Michigan Math. J. 34, 284291 (1987). MR 88j:57016

13. 148 (1988). MR 89c:57016

14. A. Ranicki, The algebraic theory of surgery II. Applications to topology, Proc. Lond. Math. Soc. 40, 193-287 (1980). MR 82f:57024b

15. - Exact sequences in the algebraic theory of surgery, Mathematical Notes 26, Princeton University Press (1981). MR 82h:57027

16. _ Algebraic L-theory and topological manifolds, Cambridge Tracts in Mathematics 102, Cambridge University Press (1992). MR 94i:57051

17. C.P. Rourke, B.J. Sanderson, On topological neighbourhoods, Compositio Math. 22, 387-425 (1970). MR 45:7720

18. C.T.C. Wall, Surgery on compact manifolds, Academic Press (1970); 2nd edition, Mathematical Monographs and Surveys 69, A.M.S. (1999) CMP 99:12; MR 55:4217 (1st ed.)

19. S. Weinberger, Nonlocally linear manifolds and orbifolds, Proc. 1994 Zürich ICM, 637-647, Birkhäuser (1995). MR 97g:57028

20. S. Weinberger, The topological classification of stratified spaces, Chicago Lectures in Mathematics, University of Chicago Press, (1994). MR 96b:57024

21. J. West, Mapping Hilbert cube manifolds to ANR's, Ann. of Math. 106, 1-18 (1977). MR 56:9534

Department of Mathematics, University of Massachusetts, Amherst, Massachusetts 01003

E-mail address: johnston@math.umass.edu

Department of Mathematics and Statistics, University of Edinburgh, Edinburgh EH9 3JZ, Scotland, UK

E-mail address: aar@maths.ed.ac.uk 